\title{
Molecular gas and star formation in Lynds 870*
}

\author{
R. Q. Mao ${ }^{1,2,3,4}$, J. Yang ${ }^{1,2}$, C. Henkel ${ }^{3}$, and Z. B. Jiang ${ }^{1,2}$ \\ 1 Purple Mountain Observatory, Chinese Academy of Sciences, Nanjing 210008, PR China \\ 2 National Astronomical Observatories, Chinese Academy of Sciences, Beijing 100012, PR China \\ 3 Max-Planck-Institut für Radioastronomie, Auf dem Hügel 69, 53121 Bonn, Germany \\ 4 Institute of Astronomy and Astrophysics, Academia Sinica, PO Box 23-141, Taipei 106, Taiwan \\ Received 17 October 2001 / Accepted 15 April 2002
}

\begin{abstract}
We present molecular line and submillimeter dust continuum observations of the Lynds 870 cloud in the vicinity of IRAS 20231+3440. Two submillimeter cores, SMM1 and SMM2, are identified mapping the $870 \mu \mathrm{m}$ dust continuum and ammonia $\left(\mathrm{NH}_{3}\right)$ emission. The total molecular mass is $\sim 70-110 M_{\odot}$. The northern core is warmer and denser than the southern one. Molecular outflows are discovered in both cores. In the northern one a significant amount of low velocity $\left(1.3-2.8 \mathrm{~km} \mathrm{~s}^{-1}\right)$ outflowing gas is found, that is hidden in the relatively broad $\mathrm{CO}$ lines but that is revealed by the narrower $\mathrm{HCO}^{+}$spectra. While IRAS $20231+3440$ is most likely the exciting star of the northern outflow, the driving source of the southern outflow is not detected by infrared surveys and must be deeply embedded in the cloud core. Large scale $(\sim 0.2 \mathrm{pc})$ infall motion is indicated by blue asymmetric profiles observed in the $\mathrm{HCO}^{+} J=3-2$ spectra. Red $K_{\mathrm{s}}$ band YSO candidates revealed by the 2MASS survey indicate ongoing star formation throughout the cloud. The calculated masses and the measured degree of turbulence are also reminiscent of clouds forming groups of stars. The excitation of the molecular lines, molecular abundances, and outflow properties are discussed. It is concluded that IRAS $20231+3440$ is a Class I object, while the southern core most likely contains a Class 0 source.
\end{abstract}

Key words. ISM: jets and outflows - ISM: molecules - stars: formation ISM: individual: Lynds 870, IRAS 20231+3440

\section{Introduction}

Stars form from collapsing dense cores of molecular clouds. Protostars arise as a result of the collapse and evolve (if their mass does not exceed a few solar masses) from Class 0 to Class I, II and III objects (André et al. 2000). The earliest stages of star formation are observationally characterized by an association with dense molecular cores, masers and molecular outflows. The dust and the gas of dense cores can be traced by submillimeter continuum and spectral line emission from tracers like $\mathrm{NH}_{3}, \mathrm{CS}$, and $\mathrm{HCO}^{+}$. Molecular outflows, usually traced in lines of the $\mathrm{CO}$ molecule with broad-line wing emission, have received considerable attention over the past two decades and over 250 outflow sources have been cataloged so far

Send offprint requests to: R. Q. Mao,

e-mail: rqmao@jets.pmo.ac.cn

* Based on observations with the 10-m Heinrich-HertzTelescope (HHT) and the MPIfR 100-m telescope at Effelsberg. The HHT is operated by the Submillimeter Telescope Observatory on behalf of Steward Observatory and the MaxPlanck-Institut für Radioastronomie. The 100-m telescope is operated by the Max-Planck-Institut für Radioastronomie.
(Fukui et al. 1993; Wu et al. 1996). Our current understanding of outflow properties and its interaction with the parental dense core is, however, heavily influenced by a few well-studied examples, and may be far from accurate.

Recently an IRAS-selected CO $J=1-0$ survey has been carried out at the $13.7 \mathrm{~m}$ mm-wave telescope of the Purple Mountain Observatory at Delingha (Yang et al. 1999, 2002; Jiang et al. 2000). A number of sources with strong CO emission and/or outflowing gas have been selected from the survey. In this work, we present detailed observations of one of the sources, IRAS 20231+3440 (hereafter I 20231).

I $20231\left(\alpha_{2000.0}=20^{\mathrm{h}} 25^{\mathrm{m}} 07^{\mathrm{s}} .0, \delta_{2000.0}=34^{\circ} 50^{\prime} 05^{\prime \prime}\right)$ is located within Lynds 870. On Palomar Observatory Sky Survey (POSS) prints, there is no optical counterpart at the position of I 20231. High extinction can be traced over a region of $15^{\prime} \times 15^{\prime}$. The infrared colour indices of I 20231 show that the source is deeply embedded (Emerson 1988). At the same time the IRAS colors satisfy the criteria introduced by Wood \& Churchwell (1989) for compact HII regions. There were some water maser detections reported (Palla et al. 1993; Brand et al. 1994), which indicate an early stage of young stellar object (YSO) formation, but 
methanol (van der Walt et al. 1996) and OH masers were not detected (Slysh et al. 1997). CS $2-1$ observations give $v_{\text {LSR }} \sim 5.4 \mathrm{~km} \mathrm{~s}^{-1}$ (Bronfman et al. 1996), which indicates a dynamical distance of $3.7 \mathrm{kpc}$ (far) or $1.0 \mathrm{kpc}$ (near distance). The optical extinction seen on POSS prints suggests that the nearer distance is more reasonable and we will take $1.0 \mathrm{kpc}$ as the distance to the source. Since all mapping observations were carried out w.r.t I 20231, we refer in the following to this as the reference position (offset $\left.\left(0^{\prime \prime}, 0^{\prime \prime}\right)\right)$.

In this paper, we present molecular line and $870 \mu \mathrm{m}$ continuum observations to trace the dense molecular gas, the outflows and the submillimeter dust continuum around I 20231. In Sect. 2, we describe our observations, and in Sects. 3 and 4 we derive physical parameters from our dust continuum and molecular line data. In Sect. 5, results from NIR photometry are studied and in Sect. 6, we discuss the global properties of star formation in the region. Conclusions are drawn in Sect. 7 .

\section{Observations}

\section{1. $\mathrm{NH}_{3}$ and $\mathrm{H}_{2} \mathrm{O}$ at Effelsberg 100-m}

The $\mathrm{NH}_{3}$ and the $22 \mathrm{GHz} \mathrm{H}_{2} \mathrm{O}$ maser observations were obtained in July 1999, April and July 2000, using the Effelsberg 100-m telescope of the Max-Plank Institute für Radioastronomie (MPIfR) equipped with a maser receiver in 1999 and a dual channel $K$-band HEMT receiver in 2000 . The typical system temperature was about $150 \mathrm{~K}-$ $200 \mathrm{~K}$ on a main beam brightness temperature scale; the half-power beam width was $\sim 40^{\prime \prime}$. The data were recorded with a 8192-channel autocorrelator. The $K$-band HEMT receiver allowed us to measure the $(J, K)=(1,1)$ to $(4,4)$ $\mathrm{NH}_{3}$ inversion lines simultaneously. The observed spectral resolution was 0.24 and $0.98 \mathrm{~km} \mathrm{~s}^{-1}$ for $\mathrm{NH}_{3}$ observations in 1999 and 2000, respectively, and about $0.13 \mathrm{~km} \mathrm{~s}^{-1}$ for the $\mathrm{H}_{2} \mathrm{O}$ maser observations. The line intensities were calibrated relative to the continuum of NGC 7027, for which we assume a flux density of $5.86 \mathrm{Jy}$, corresponding to a main beam brightness temperature of $8.1 \mathrm{~K}$. Pointing was checked every hour on nearby continuum sources and was found to be stable within $10^{\prime \prime}$. The calibration accuracy is estimated to be $\pm 20 \%$.

\section{2. $\mathrm{mm}$ and sub-mm line observations at HHT $10 \mathrm{~m}$}

Molecular line emission of $\mathrm{CO}, \mathrm{CS}, \mathrm{HCO}^{+}$and some of their rare isotopes was observed with the 10-m Heinrich Hertz Telescope (HHT; see Baars et al. 1999) on Mt. Graham in Southern Arizona during three sessions in April 1999, January 2000 and May 2000. The receivers were sensitive to both sidebands. A dual channel $345 \mathrm{GHz}$ SIS receiver was used for the $J=3-2$ transitions of ${ }^{12} \mathrm{CO}$ and its isotopes, while all the $\mathrm{mm}$-wave lines were observed with a single channel $230 \mathrm{GHz}$ SIS receiver. For the ${ }^{12} \mathrm{CO}$ mapping we used the spectral line On-TheFly (OTF) method, in which we scanned the telescope at a rate of about $1^{\prime \prime}$ per second. The off position was located at $\Delta \alpha=1^{\circ}$ west of I 20231 and was free of $\mathrm{CO}$ emission. The most central region $\left(\sim 1^{\prime}-2^{\prime}\right)$ around I 20231 was also mapped in ${ }^{13} \mathrm{CO}, \mathrm{C}^{18} \mathrm{O}, \mathrm{C}^{17} \mathrm{O}, \mathrm{HCO}^{+}$, $\mathrm{H}^{13} \mathrm{CO}^{+}$and CS with standard position switching (off position at $\left.\Delta \alpha=20^{\prime}\right)$. The $J=5-4$ lines of three rare CS isotopes, ${ }^{12} \mathrm{C}^{34} \mathrm{~S},{ }^{12} \mathrm{C}^{33} \mathrm{~S}$ and ${ }^{13} \mathrm{C}^{32} \mathrm{~S}$, were obtained towards the central position of I 20231.

For the ${ }^{12} \mathrm{CO}$ observations we used two 2048-channel $1 \mathrm{GHz}$ Acousto-Optical Spectrometers (AOS) as backends, with a mean channel spacing of $917 \mathrm{KHz}$ corresponding to $\sim 0.78$ and $1.19 \mathrm{~km} \mathrm{~s}^{-1}$ at 345 and $230 \mathrm{GHz}$, respectively. A 256-channel filter bank with a channel spacing of $250 \mathrm{kHz}$, providing higher spectral resolution, was used simultaneously for other molecular lines. The beam widths were about 22 and $33^{\prime \prime}$ for 345 and $230 \mathrm{GHz}$ respectively.

All results displayed are given on a main beam brightness temperature scale $\left(T_{\mathrm{MB}}\right)$, which is related to $T_{\mathrm{A}}^{*}$ via $T_{\mathrm{MB}}=T_{\mathrm{A}}^{*}\left(F_{\text {eff }} / B_{\text {eff }}\right)$ (see Downes 1989$)$. Main beam efficiencies, $B_{\text {eff }}$, were 0.5 and 0.78 at 345 and $230 \mathrm{GHz}$, as obtained from measurements of Saturn. Forward hemisphere efficiencies, $F_{\text {eff }}$, were 0.9 and 0.95 , respectively. For ${ }^{12} \mathrm{CO}, \chi$ Cyg was used for both pointing and absolute line intensity calibration. The rms pointing uncertainty was found to be $\sim 5^{\prime \prime}$. Line intensities were found to be accurate within $20 \%$ when compared to the $J=3-2$ line of CO of Stanek et al. (1995) and the $J=2-1$ line of Loup et al. (1993). For other lines, Orion-KL was observed as a secondary calibrator and the line survey results by Sutton et al. (1985) and Blake et al. (1986) near $230 \mathrm{GHz}$ and Schilke et al. (1997) near $345 \mathrm{GHz}$ were used for comparison. Again calibration errors should not exceed $20 \%$.

\section{3. $870 \mu m$ continuum observations}

$870 \mu \mathrm{m}$ dust continuum emission was imaged with the HHT 19 channel bolometer array. The array covers a hexagonal $200^{\prime \prime}$ field of view. The beam width of the HHT is about $22^{\prime \prime}$ at $870 \mu \mathrm{m}$. Maps were taken using the OTF method with a beam throw of $120^{\prime \prime}$ and a scanning speed of $8^{\prime \prime}$ per second. Five OTF maps, with three coverages $\left(400^{\prime \prime} \times 300^{\prime \prime}\right.$ in size $)$ centered at I 20231 and another two coverages $\left(320^{\prime \prime} \times 260^{\prime \prime}\right)$ centered at $\left(0^{\prime \prime},-180^{\prime \prime}\right)$ relative to I 20231 were finally combined by mosaicing. The average rms noise level is about $90 \mathrm{mJy}$ in the combined map. All maps were calibrated using $870 \mu \mathrm{m}$ skydips and the standard calibrators Uranus and G 34.3+0.2, and a peak flux density of $60 \mathrm{Jy}^{\mathrm{beam}}{ }^{-1}$ was assumed for the latter one. At $870 \mu \mathrm{m}$, the typical atmospheric opacity was about 0.4 during the observations. The calibration accuracy should be better than $\pm 20 \%$.

\section{Dust continuum emission}

A $870 \mu \mathrm{m}$ continuum image of $\mathrm{L} 870$ is presented in Fig. 1. The map reveals two submillimeter cores (SMM), the northern one, SMM1, peaked at $\left(7^{\prime \prime},-7^{\prime \prime}\right)$ with a peak flux density of $1.67 \mathrm{Jy}$ beam $^{-1}$, directly associated with I 20231 


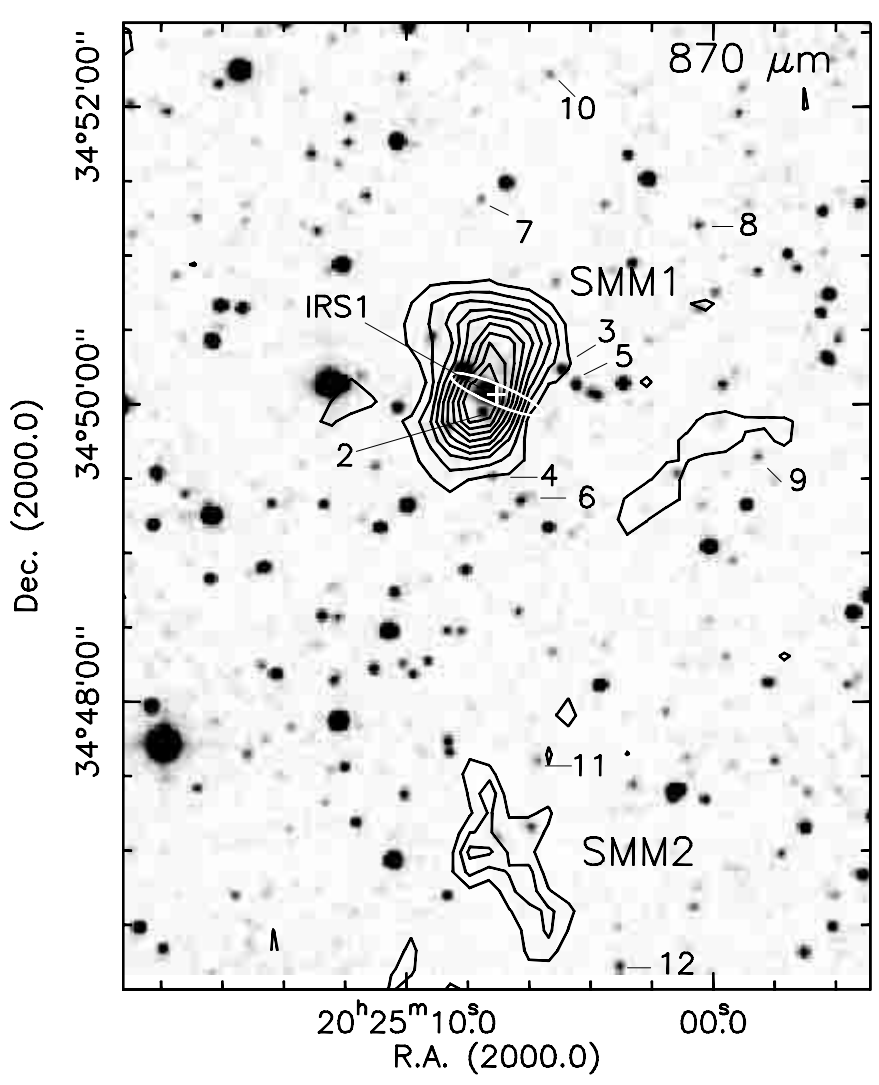

Fig. 1. HHT $870 \mu \mathrm{m}$ continuum image overlaid on a 2MASS$K_{\mathrm{s}}$ image. Contour levels are $15 \%(\sim 3 \sigma)$ to $95 \%$ of the peak flux ( $1.7 \mathrm{Jy})$ by steps of $10 \%$. The position of the IRAS point source I 20231 is indicated by the white cross and the ellipse is the IRAS $1 \sigma$ positional uncertainty. Also shown are the 12 red $K_{\mathrm{s}}$ sources IRS1, 2 to 12, which are discussed in Sect. 5.

and the southern one, SMM2, peaked at $\left(12^{\prime \prime},-188^{\prime \prime}\right)$ with a peak flux density of $0.60 \mathrm{Jy}^{\text {beam }}{ }^{-1}$, not associated with any IRAS point source. The northern core region is elongated along a position angle of $P A \sim-20^{\circ}$ with a deconvolved half-power size of $17^{\prime \prime}$ by $50^{\prime \prime}$ (0.08 pc by $0.25 \mathrm{pc}$ ), while the southern core region looks more irregular. Also shown in the map is a filament southwest of I 20231. This is however only a $\sim 3 \sigma$ detection, and deeper images are needed to confirm its existence. The integrated flux densities of the northern, the southern and the filament regions are $6.5 \pm 1.1,1.7 \pm 0.5$ and $0.7 \pm 0.2 \mathrm{Jy}$, respectively.

The masses of the clouds can be estimated via $M=$ $F_{\nu} D^{2} / \kappa_{\nu} B_{\nu}\left(T_{\mathrm{d}}\right)$, where $F_{\nu}$ is the flux density, $D$ denotes the distance towards the cloud, $B_{\nu}\left(T_{\mathrm{d}}\right)$ is the Plank function for temperature $T_{\mathrm{d}}$, and $\kappa_{\nu}$ is the dust opacity per unit gas and dust mass. Various values of $\kappa_{\nu}$ for different regions can be found in the literature (e.g. Draine \& Lee 1984; Hildebrand 1983; Preibisch et al. 1993; Ossenkopf \& Henning 1994; Krügel \& Siebenmorgen 1994). We adopt here the value $\kappa_{870 \mu \mathrm{m}}=0.01 \mathrm{~cm}^{2} \mathrm{~g}^{-1}$, using the Hildebrand (1983) assumptions, with $\beta=2$ ( $\beta$ : exponent in the frequency dependence of the dust grain emissivity). For the southern dust core, we apply a dust temperature of $12 \mathrm{~K}$, a typical value for star forming cores
(Williams et al. 1999; Bacmann et al. 2000). For the northern core, we take a higher dust temperature of $30 \mathrm{~K}$, as indicated by the color temperature of $\sim 33 \mathrm{~K}$ derived from 60 and $100 \mu \mathrm{m}$ dust emission (Henning et al. 1990). Total masses traced by the dust are then $33.9 \pm 6.3,40.0 \pm 11.8$ and $16.5 \pm 4.7 M_{\odot}$ for the northern core, the southern core and the filament, respectively. Note that the masses derived in this way can be linearly scaled to another value of $\kappa_{870 \mu \mathrm{m}}$ if desired; moreover masses are sensitive to the temperature and a higher temperature would decrease the calculated mass inversely proportional.

The beam averaged $\left(22^{\prime \prime}\right)$ peak column density, derived from the peak flux density of $1.67 \mathrm{Jybeam}^{-1}$, is $9.6 \times 10^{22} \mathrm{~cm}^{-2}$, which gives a corresponding peak extinction of $A_{\mathrm{v}} \sim 100\left(N\left(\mathrm{H}_{2}\right)=A_{\mathrm{v}} \times 9.5 \times 10^{20} \mathrm{~cm}^{-2}\right.$, Bohlin et al. 1978). The beam averaged peak column density for a different beam size can be calculated by smoothing the image to the corresponding beam size, assuming a Gaussian shape of the beam. Column densities of 7.5, 7.0, 6.3 and $5.5 \times 10^{22} \mathrm{~cm}^{-2}$ are derived for beam sizes of $29,32,35$ and $40^{\prime \prime}$, respectively. To estimate molecular abundances assuming they trace the same volume as the $870 \mu \mathrm{m}$ dust continuum emission, these values will be used in the following.

\section{Molecular line emission}

Results derived from our molecular line observations are summarized in Table 1, which lists: molecular line, rest frequency, half power beam width (HPBW) at the corresponding frequency, main beam brightness temperature, Local Standard of Rest (LSR) velocity, full width to half power (FWHP) line width, integrated line intensity, estimated optical depth averaged over the line profile, and offset position relative to I 20231. The $\Delta v_{\text {int }}$ values presented in Col. 6 are intrinsic line widths that were deconvolved by the spectral resolution. The $\bar{\tau}$ value given in Col. 8 is estimated from the ratio of integrated intensities of the same transition in two different isotopes, assuming isotope ratios of $\left[{ }^{13} \mathrm{C}\right]:\left[{ }^{12} \mathrm{C}\right]=1: 69.7,\left[{ }^{18} \mathrm{O}\right]:\left[{ }^{17} \mathrm{O}\right]:\left[{ }^{16} \mathrm{O}\right]=5.5: 1: 2856$ (Wilson \& Rood 1994), and $\left[{ }^{34} \mathrm{~S}\right]:\left[{ }^{32} \mathrm{~S}\right]=31.3$ (Chin et al. 1996) for a galactocentric distance of $8.2 \mathrm{kpc}$. In the case of $\mathrm{NH}_{3}$, the peak optical depth of the main group of hyperfine components is given. We assume local thermodynamic equilibrium (LTE) at an excitation temperature $T_{\text {ex }}$ and identical beam filling factors (unity) throughout the following analysis. For the line emission from linear, rigid rotor molecules such as $\mathrm{CO}, \mathrm{CS}, \mathrm{HCO}^{+}$and their isotopes, the beam averaged column density in $\mathrm{cm}^{-2}$ is given by

$$
\begin{aligned}
\bar{N}= & 10^{5} \times \frac{3 k^{2}}{4 h \pi^{3} \mu^{2} \nu^{2}} \exp \left(\frac{h \nu J}{2 k T_{\text {ex }}}\right) \frac{T_{\text {ex }}+h \nu / 6 k(J+1)}{e^{-h \nu / k T_{\text {ex }}}} \\
& \times \frac{\bar{\tau}}{1-e^{-\bar{\tau}}} \int T_{\mathrm{MB}} \mathrm{d} v
\end{aligned}
$$

(Scoville et al. 1986), where $\int T_{\mathrm{MB}} \mathrm{d} v$ is the integrated intensity in $\mathrm{K} \mathrm{km} \mathrm{s}^{-1}$ of the $J+1 \rightarrow J$ transitions with frequency $\nu(\mathrm{Hz})$ and optical depth $\bar{\tau} . k\left(\mathrm{erg} \mathrm{k}^{-1}\right)$ and $h$ 
Table 1. Observed molecular line parameters*.

\begin{tabular}{|c|c|c|c|c|c|c|c|c|}
\hline Line & $\begin{array}{r}\text { Frequency } \\
(\mathrm{MHz})\end{array}$ & $\begin{array}{l}\text { HPBW } \\
\text { (arcsec) }\end{array}$ & $\begin{array}{c}T_{\mathrm{MB}} \\
(\mathrm{K})\end{array}$ & $\begin{array}{c}v_{\mathrm{LSR}} \\
\left(\mathrm{km} \mathrm{s}^{-1}\right) \\
\end{array}$ & $\begin{array}{c}\Delta v_{\text {int }} \\
\left(\mathrm{km} \mathrm{s}^{-1}\right)\end{array}$ & $\int_{\left(\mathrm{K} \mathrm{km} \mathrm{s}^{-1}\right)} T_{\mathrm{MB}} \mathrm{d} v$ & $\bar{\tau}$ & $\begin{array}{c}\text { Offset } \\
\left({ }^{\prime \prime},{ }^{\prime \prime}\right)\end{array}$ \\
\hline${ }^{12} \mathrm{C}^{16} \mathrm{O}(3-2)$ & 345795.975 & 22 & & & & $64.8(.28)$ & & $(0,0)$ \\
\hline${ }^{12} \mathrm{C}^{16} \mathrm{O}(2-1)$ & 230537.990 & 33 & & & & $82.8(.32)$ & & $(0,0)$ \\
\hline${ }^{13} \mathrm{C}^{16} \mathrm{O}(3-2)$ & 330587.957 & 23 & $5.37(.12)$ & $6.00(.02)$ & $3.45(.04)$ & $19.8(.12)$ & $3.78(1.54)$ & $(0,0)$ \\
\hline${ }^{13} \mathrm{C}^{16} \mathrm{O}(2-1)$ & 220398.686 & 35 & $5.76(.12)$ & $6.10(.02)$ & $3.68(.03)$ & $22.6(.19)$ & $3.84(1.00)$ & $(0,0)$ \\
\hline${ }^{12} \mathrm{C}^{18} \mathrm{O}(3-2)$ & 329330.570 & 23 & $2.20(.15)$ & $6.17(.06)$ & $3.19(.17)$ & $8.03(.30)$ & $0.50(0.21)$ & $(0,0)$ \\
\hline${ }^{12} \mathrm{C}^{18} \mathrm{O}(2-1)$ & 219560.360 & 35 & $3.31(.14)$ & $6.24(.03)$ & $2.60(.06)$ & $9.26(.20)$ & $0.51(0.13)$ & $(0,0)$ \\
\hline${ }^{12} \mathrm{C}^{17} \mathrm{O}(2-1)$ & 224714.368 & 35 & $0.45(.06)$ & $6.60(.15)$ & $1.65(.45)$ & $1.41(.15)$ & ... & $(0,0)$ \\
\hline \multirow[t]{2}{*}{${ }^{12} \mathrm{C}^{32} \mathrm{~S}(5-4)$} & 244935.643 & 32 & $1.67(.14)$ & $6.26(.05)$ & $3.23(.13)$ & $5.77(.15)$ & $1.81(0.84)$ & $(0,0)$ \\
\hline & & & $0.51(.13)$ & $6.08(.10)$ & $2.72(.24)$ & $1.49(.11)$ & & $(0,-180)$ \\
\hline${ }^{12} \mathrm{C}^{34} \mathrm{~S}(5-4)$ & 241016.194 & 32 & $0.13(.02)$ & $6.72(.26)$ & $2.84(.55)$ & $0.39(.07)$ & $0.06(0.03)$ & $(0,0)$ \\
\hline${ }^{12} \mathrm{C}^{33} \mathrm{~S}(5-4)$ & 242913.680 & 32 & $<0.06$ & & & & & $(0,0)$ \\
\hline${ }^{13} \mathrm{C}^{32} \mathrm{~S}(5-4)$ & 231220.996 & 35 & $<0.06$ & & & & & $(0,0)$ \\
\hline $\mathrm{HCO}^{+}(3-2)$ & 267577.625 & 29 & & & & $15.8(.49)$ & $3.56(3.40)$ & $(0,0)$ \\
\hline $\mathrm{H}^{13} \mathrm{CO}^{+}(3-2)$ & 260255.480 & 29 & $0.41(.07)$ & $6.54(.08)$ & $1.85(.21)$ & $0.81(.08)$ & $0.05(0.05)$ & $(0,0)$ \\
\hline \multirow[t]{2}{*}{$\mathrm{NH}_{3}(1,1)$} & 23694.496 & 40 & $3.80(.10)$ & $6.17(.02)$ & $1.95(.05)$ & $25.2(.10)$ & $1.30(0.10)$ & $(0,0)$ \\
\hline & & & $2.50(.10)$ & $5.78(.03)$ & $1.35(.09)$ & $10.6(.10)$ & & $(0,-180)$ \\
\hline \multirow[t]{2}{*}{$\mathrm{NH}_{3}(2,2)$} & 23722.631 & 40 & $1.30(.10)$ & $6.07(.07)$ & $2.44(.20)$ & $5.20(.10)$ & & $(0,0)$ \\
\hline & & & $0.50(.10)$ & $5.95(.13)$ & $1.35(.16)$ & $0.95(.30)$ & & $(0,-180)$ \\
\hline $\mathrm{NH}_{3}(3,3)$ & 23870.130 & 40 & $0.48(.02)$ & $6.33(.07)$ & $3.50(.22)$ & $1.94(.07)$ & & $(0,0)$ \\
\hline $\mathrm{NH}_{3}(4,4)$ & 24139.417 & 40 & $0.08(.01)$ & $6.62(.36)$ & $3.77(.79)$ & $0.34(.06)$ & & $(0,0)$ \\
\hline
\end{tabular}

* Columns 4 to 7 are from Gaussian fitting, except for $\mathrm{CO}(3-2),(2-1)$ and $\mathrm{HCO}^{+}(3-2)$ for which only total integrated intensities are given due to self-absorption features in the spectra. Column 8 gives the "averaged" optical depth over the line profile (see text). Offset positions relative to I 20231 are presented in the last column. Note that Col. 6 gives the intrinsic full width to half power (FWHP) line width which has been deconvolved with the corresponding spectral resolution. Hyperfine structure has been taken into account fitting the $\mathrm{C}^{17} \mathrm{O}(2-1)$ and $\mathrm{NH}_{3}$ lines. Upper limits to the main beam brightness temperatures are $3 \sigma$. The errors (standard deviations) of all these parameters are given in parentheses.

(erg s) denote the Boltzmann constant and the Planck constant, respectively, and $\mu$ (esu cm) is the permanent dipole moment. For $\mathrm{NH}_{3}$ column densities, see Sect. 4.1.

\section{1. $\mathrm{NH}_{3}$ data}

Figure 2 illustrates the distribution of integrated intensity of the $\mathrm{NH}_{3}(1,1)$ main group of lines. The whole region can be divided into three subregions, with two cores located close to the two $870 \mu \mathrm{m}$ peaks and a ridge in between. Such a morphology generally resembles the twin core system defined by Jijina et al. (1999) in their extensive study of nearby $\mathrm{NH}_{3}$ dense cores. The northern core has an associated IRAS source (I 20231), while the southern one does not. The spatial structure of the ammonia cores more or less follows the $870 \mu \mathrm{m}$ dust morphology. With a deconvolved half-power size of $35^{\prime \prime}$ by $57^{\prime \prime}$ ( $0.17 \mathrm{pc}$ by $0.28 \mathrm{pc})$, the northern core is more centrally peaked than the southern one, which has a deconvolved size of about $75^{\prime \prime}(0.36 \mathrm{pc})$. To the northwest of the ridge emission, there is a spur structure, which matches the weak $870 \mu \mathrm{m}$ filament, suggesting that the continuum feature is real.

While $(1,1)$ and $(2,2)$ line emission of ammonia is fairly extended in the region, $(3,3)$ emission is more concentrated around I 20231 and $(4,4)$ emission is only detected towards I 20231 itself with high signal to noise ratio $(S / N)$. Observed parameters of the $(1,1)$ to $(4,4)$ lines are listed in Table 1 (see attached spectra in Fig. 2).

All positions detected in $(1,1)$ and $(2,2)$ emission were analysed with the usual technique (see Harju et al. 1993). The rotation temperatures $T_{\text {rot }}$, the column densities $N\left(\mathrm{NH}_{3}\right)$, and the number densities $n\left(\mathrm{H}_{2}\right)$ at the two peak positions $\left(0^{\prime \prime}, 0^{\prime \prime}\right)$ and $\left(0^{\prime \prime},-180^{\prime \prime}\right)$ are calculated to be 15.8 and $12.9 \mathrm{~K}, 1.45 \times 10^{15}$ and $7.8 \times 10^{14} \mathrm{~cm}^{-2}$, $1.9 \times 10^{4}$ and $3.5 \times 10^{3} \mathrm{~cm}^{-3}$, respectively. However, $T_{\text {rot }}$ derived from $(1,1)$ and $(2,2)$ emission usually tends to underestimate the gas kinetic temperature $T_{\text {kin }}$, which can be better approached by a $T_{\text {rot }}$ estimated from transitions with higher excitation (Walmsley \& Ungerechts 1983; Danby et al. 1988). We thus alternatively apply a rotation diagram for a better determination of $T_{\text {kin }}$ for the $\left(0^{\prime \prime}, 0^{\prime \prime}\right)$ position where a good $S / N$ ratio $(4,4)$ spectrum was taken. Assuming a single $T_{\text {rot }}$ for the populations in all metastable levels, a best fit gives a $T_{\text {rot }}=31.8 \pm 6.2 \mathrm{~K}$ from the $(1,1),(2,2)$ and $(4,4)$ transitions of para- $\mathrm{NH}_{3}$. We can thus safely conclude a $T_{\text {kin }} \geq 25 \mathrm{~K}$ for the dense gas within a region $40^{\prime \prime}$ in diameter around I 20231.

From the beam averaged $\mathrm{H}_{2}$ column density for a $40^{\prime \prime}$ area towards I 20231 (see Sect. 3), we can estimate the $\mathrm{NH}_{3}$ fractional abundance $\chi\left(\mathrm{NH}_{3}\right)=\left[\mathrm{NH}_{3}\right] /\left[\mathrm{H}_{2}\right] \sim$ $2.6 \times 10^{-8}$. The masses are then 43,53 and $12 M_{\odot}$ for 


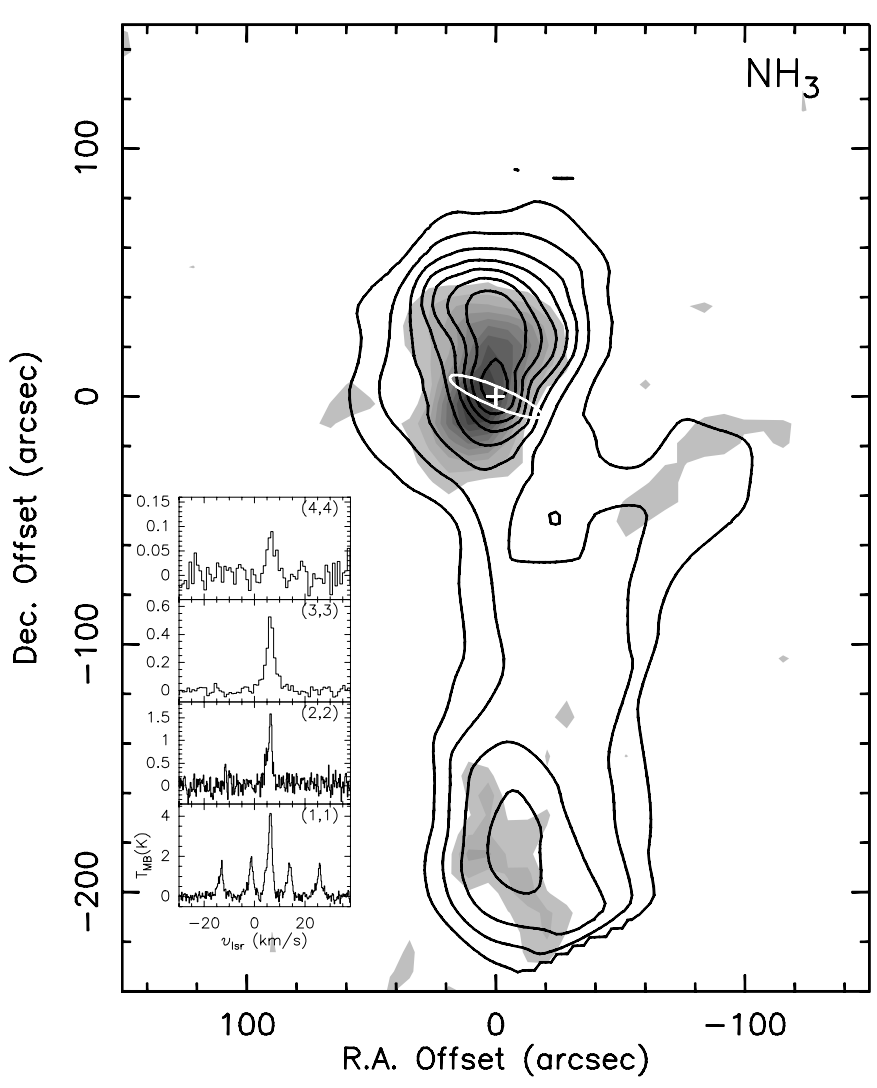

Fig. 2. Effelsberg $\mathrm{NH}_{3}$ map (contours) of the integrated $(1,1)$ main group of lines overlaid on the $870 \mu \mathrm{m}$ continuum image (grey). Contour levels are $20 \%$ to $90 \%$ of the peak value $\left(9.5 \mathrm{~K} \mathrm{~km} \mathrm{~s}^{-1}\right)$ with a spacing of $10 \% . \mathrm{NH}_{3}(1,1)$ to $(4,4)$ spectra taken at the I 20231 position are attached. Note that the spectral resolution is $0.24 \mathrm{~km} \mathrm{~s}^{-1}$ for the $(1,1)$ and $(2,2)$ lines and $0.98 \mathrm{~km} \mathrm{~s}^{-1}$ for the $(3,3)$ and $(4,4)$ lines. For the white cross and the error ellipse, see Fig. 1.

the northern core, southern core and the ridge including the spur, respectively. These masses agree well with those derived from submillimeter dust emission. The total mass of the whole region is therefore $\sim 110 M_{\odot}$. The virial masses derived from the line width and core size are 113 and $52 M_{\odot}$ for the northern and southern core, respectively. While the southern core gives a virial mass very close to the masses derived from dust and $\mathrm{NH}_{3}$ emission, the northern core shows a significant inconsistency. This may be due to line broadening by outflowing gas which will be discussed below.

\subsection{CO data}

\subsubsection{Ambient $\mathrm{CO}$ emission}

Spectra of selected $\mathrm{CO}$ isotopes taken at the position of I 20231 are shown in Fig. 3. The ${ }^{12} \mathrm{CO} J=3-2$ and $2-1$ spectra show similar line shapes, with broad-line wings over more than $40 \mathrm{~km} \mathrm{~s}^{-1}$ and a deep dip at $\sim 6 \mathrm{~km} \mathrm{~s}^{-1}$. Since the off position was carefully chosen to be free of emission and because of the lineshapes of the ${ }^{13} \mathrm{CO}, \mathrm{C}^{18} \mathrm{O}$ and $\mathrm{C}^{17} \mathrm{O}$ profiles (see below), we believe that the dip

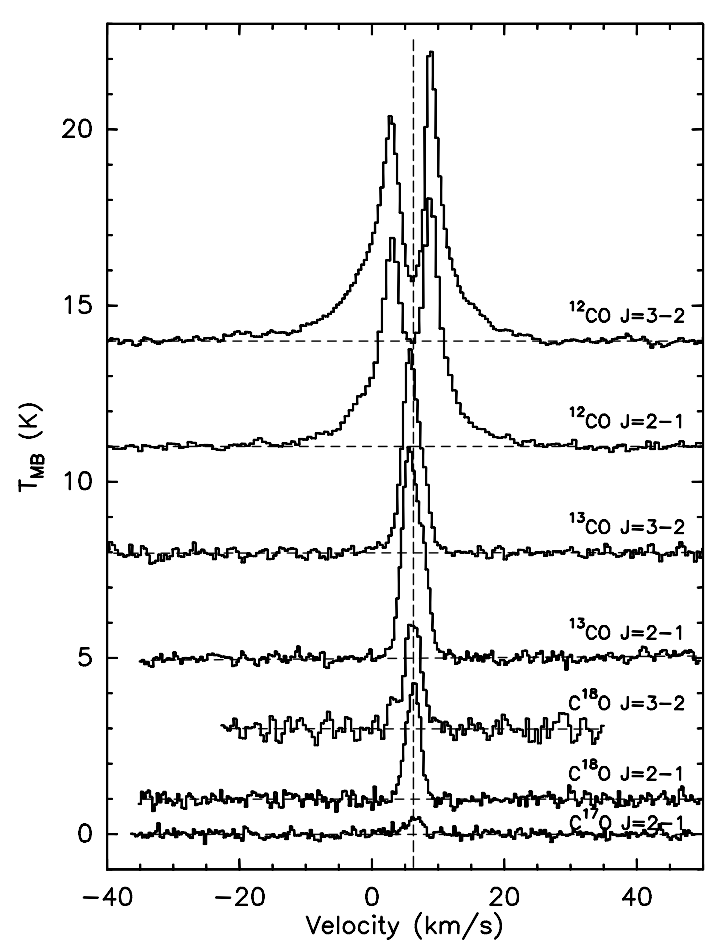

Fig. 3. Selected HHT spectra of $\mathrm{CO}$ and its isotopes towards I 20231. The dashed vertical line marks the systemic velocity of $v_{\mathrm{LSR}}=6.3 \mathrm{~km} \mathrm{~s}^{-1}$.

feature is caused by self-absorption. The self-absorption between 3 and $8 \mathrm{kms}^{-1}$, with a $T_{\mathrm{MB}}$ of about $3 \mathrm{~K}$ and $2 \mathrm{~K}$ in $J=2-1$ and $3-2$, respectively, requires a consistent excitation temperature of $T_{\mathrm{ex}} \sim 7.5 \mathrm{~K}$, which is likely the temperature of the outermost layer of the cloud. The ${ }^{13} \mathrm{CO}, \mathrm{C}^{18} \mathrm{O}$ and $\mathrm{C}^{17} \mathrm{O}$ lines are peaked at the velocity of the ${ }^{12} \mathrm{CO}$ dip (marked as a dashed vertical line in Fig. 3), with a $v_{\mathrm{LSR}}$ of $6.3 \mathrm{~km} \mathrm{~s}^{-1}$, very close to that of our $\mathrm{NH}_{3}$ lines. From the ratio of peak temperatures of the ${ }^{13} \mathrm{CO}$ and $\mathrm{C}^{18} \mathrm{O}$ lines, assuming a $\left[{ }^{13} \mathrm{CO}\right] /\left[\mathrm{C}^{18} \mathrm{O}\right]$ abundance ratio of 7.5 (Wilson \& Rood 1994), we find an LTE excitation temperature of $10 \mathrm{~K}$ for the $J=2-1$ and $11.5 \mathrm{~K}$ for the $J=3-2$ emission, and moderate optical depths of 0.96 and 0.8 at the peak velocity for the $J=2-1$ and $3-2$ transitions of $\mathrm{C}^{18} \mathrm{O}$, respectively. We adopt an excitation temperature of $10 \mathrm{~K}$ for the ambient molecular gas in the following calculations. Estimated from the ratio of integrated intensities of ${ }^{13} \mathrm{CO}$ and $\mathrm{C}^{18} \mathrm{O}$, we find $\bar{\tau} \sim 0.5$ for the $J=3-2$ and $2-1$ line profiles of $\mathrm{C}^{18} \mathrm{O}$. After applying a $\tau$-correction (multiply by a factor of $\left.\bar{\tau} /\left(1-e^{-\bar{\tau}}\right)\right)$, we estimate LTE $\mathrm{C}^{18} \mathrm{O}$ column densities of $1.4 \times 10^{16}$ and $7.3 \times 10^{15} \mathrm{~cm}^{-2}$ for the inner $22^{\prime \prime}$ and $33^{\prime \prime}$ of the cloud. These lead to self-consistent fractional abundances of $\left[\mathrm{C}^{18} \mathrm{O}\right] /\left[\mathrm{H}_{2}\right] \sim 1.5 \times 10^{-7}$ and $1.2 \times 10^{-7}$, which are in good agreement with the "typical" value of $1.7 \times 10^{-7}$ (Frerking et al. 1982).

${ }^{13} \mathrm{CO}$ and $\mathrm{C}^{17} \mathrm{O}$ were also mapped in the central part of the northern core (see panels a) and b) of Fig. 4). $\mathrm{C}^{17} \mathrm{O}$ is believed to be optically thin and therefore can be used for mass estimation. The peak LTE column density of $\mathrm{C}^{17} \mathrm{O}$ is calculated to be $8.6 \times 10^{14} \mathrm{~cm}^{-2}$, which gives 


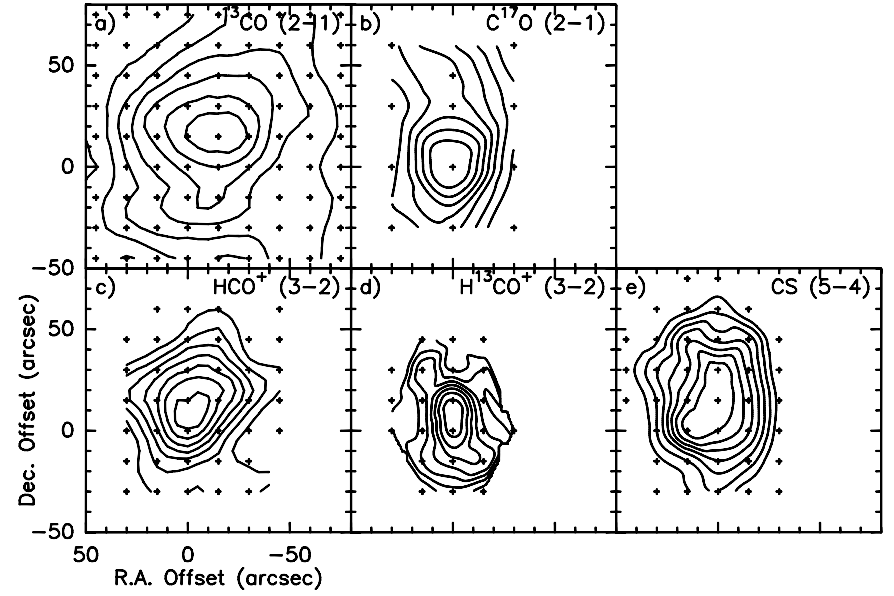

Fig. 4. Contour maps of a) ${ }^{13} \mathrm{CO}(2-1)$, b) $\mathrm{C}^{17} \mathrm{O}(2-1)$, c) $\mathrm{HCO}^{+}(3-2)$, d) $\mathrm{H}^{13} \mathrm{CO}^{+}(3-2)$ and e) $\mathrm{CS}(5-4)$ taken around I 20231. The contour levels are $30 \%$ to $90 \%$ by $10 \%$ of the peak values, which are $33.3,1.4,17.2,1.2$ and $6.5 \mathrm{~K} \mathrm{~km} \mathrm{~s}^{-1}$, respectively. Line names are labeled at the right top corner of each panel. The plus signs mark the observed positions.

a fractional $\mathrm{C}^{17} \mathrm{O}$ abundance of $1.4 \times 10^{-8}$. Using the integrated emission over the mapped region, we estimate a quiescent molecular cloud mass associated with $\mathrm{C}^{17} \mathrm{O}$ emission to be $\sim 50 M_{\odot}$, comparable to the mass derived from $\mathrm{NH}_{3}$. Note that this mass is only a lower limit due to the incompleteness of the map. The deconvolved size estimated from the $\mathrm{C}^{17} \mathrm{O}$ emission is about $40^{\prime \prime}$ by $75^{\prime \prime}$ $(0.2 \mathrm{pc}$ by $0.36 \mathrm{pc})$, slightly larger than those estimated from the $870 \mu \mathrm{m}$ dust emission and the $(1,1)$ line emission of $\mathrm{NH}_{3}$.

\subsubsection{The outflows}

Broad-line wing emission has been detected in both $\mathrm{CO}$ $J=2-1$ and 3-2 transitions toward the central region in $\mathrm{L} 870$. The ${ }^{12} \mathrm{CO}$ wing emission, determined from a comparison of $\mathrm{C}^{18} \mathrm{O}$ and ${ }^{12} \mathrm{CO}$ profiles, is defined by velocities redder than $9 \mathrm{~km} \mathrm{~s}^{-1}$ or bluer than $3 \mathrm{~km} \mathrm{~s}^{-1}$. Since CO $J=2-1$ and 3-2 maps of the outflowing material show very similar bipolar structures, we present in Fig. 5 only the higher angular resolution $\mathrm{CO} J=3-2$ data. The blue$\left(-12\right.$ to $\left.3 \mathrm{~km} \mathrm{~s}^{-1}\right)$ and the red-shifted (9 to $\left.19 \mathrm{~km} \mathrm{~s}^{-1}\right)$ lobes are shown in solid and dashed contours, respectively. Note that the integral of the redshifted wing emission was intentionally truncated to avoid contamination from a separate velocity component at about $20 \mathrm{~km} \mathrm{~s}^{-1}$, which appears in three attached spectra at the right side of Fig. 5.

Being centered on I 20231, the outflow lobes are asymmetric and show some evidence of clumpiness, especially in the red lobe. There is some overlap between red and blueshifted emission. Interestingly, close to the southern dust continuum peak, a pair of weak blue- and red-shifted lobe is also detected, indicative of ongoing star formation activity in this region too. Velocity channel maps (Fig. 6) and a position velocity map along the declination axis (Fig. 7) support this view. The blue-shifted lobe is, however, not fully covered by our map.

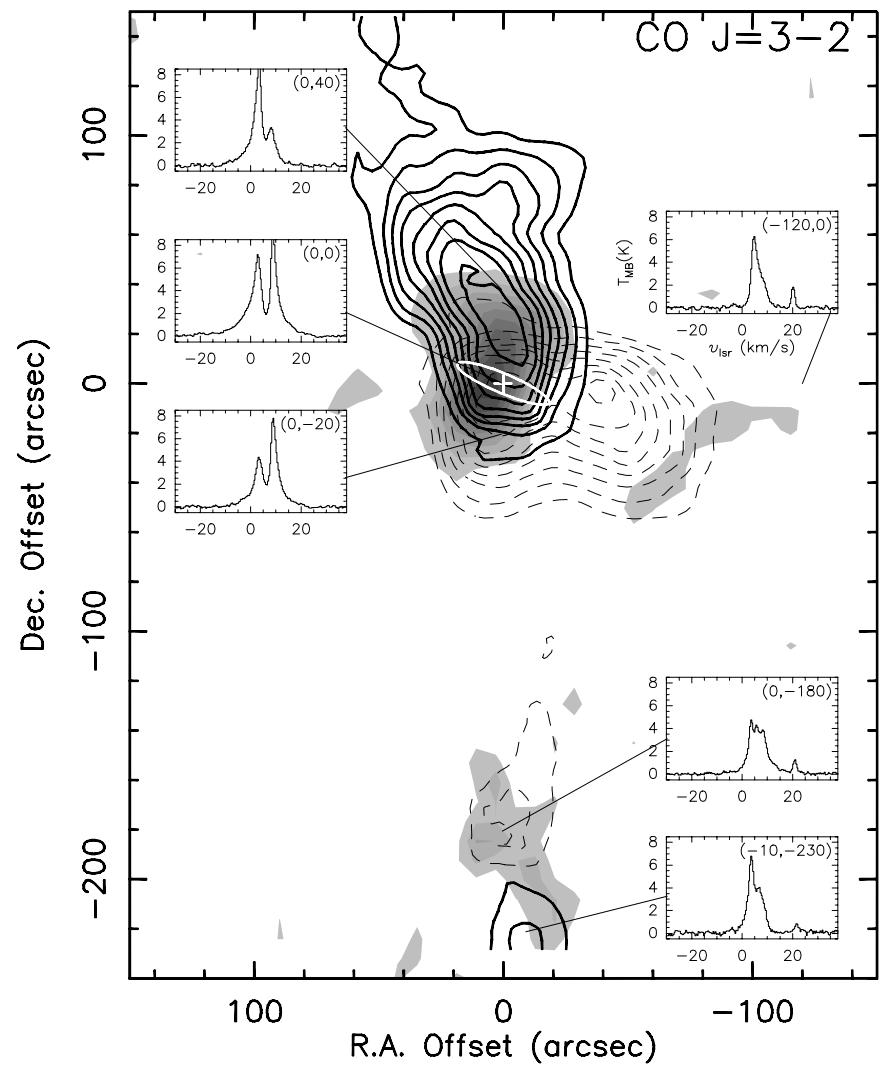

Fig. 5. Bipolar outflow of CO $J=3-2$ (contours) overlaid on our $870 \mu \mathrm{m}$ continuum image (grey). The red-shifted (dashed line) emission is integrated from 9 to $19 \mathrm{~km} \mathrm{~s}^{-1}$, and the blueshifted (solid line) emission is integrated from -12 to $3 \mathrm{~km} \mathrm{~s}^{-1}$. Contour levels are $30 \%, 40 \%, \ldots, 90 \%, 99 \%$ of the peak values, which are 35.2 (blue) and $28.8 \mathrm{~K} \mathrm{~km} \mathrm{~s}^{-1}$ (red), respectively. Spectra of selected positions (with offsets from I 20231 in $\operatorname{arcsec)}$ are also shown.

Figure 6 presents a series of velocity maps in steps of $3 \mathrm{~km} \mathrm{~s}^{-1}$. We have skipped the emission between $3 \mathrm{~km} \mathrm{~s}^{-1}$ and $9 \mathrm{~km} \mathrm{~s}^{-1}$ that is affected by self-absorption. As the maps show, the blue emission in the northern region forms a well-collimated lobe with I 20231 near one end and extends northeast, while the red emission shows two peaks, one at I 20231 and the other $40^{\prime \prime}$ to the west. There are two clumps emerging in the 18 to $21 \mathrm{~km} \mathrm{~s}^{-1}$ channel map (right bottom panel in Fig. 6), with a more compact one located about $2^{\prime}$ west of I 20231 and another one centered around the southern dust continuum peak SMM2. They are most likely cloudlets along the light-of-sight towards L 870, but the kinematic distances could not be estimated since their characteristic velocities fall into a forbidden zone. As shown in Fig. 7, the strong self-absorption at ambient velocities in the northern core appears between two bright emission peaks at about 3 and $8.5 \mathrm{~km} \mathrm{~s}^{-1}$.

The mean optical depth in the line wings, calculated from ${ }^{12} \mathrm{CO}$ to ${ }^{13} \mathrm{CO}$ line ratios, is $\sim 3.0$ for blue- $(-12$ to $+3 \mathrm{~km} \mathrm{~s}^{-1}$ ) and $\sim 2.0$ for redshifted (9 to $19 \mathrm{kms}$ ) wing emission. From the ratio of CO $J=3-2$ and 2-1 brightness temperatures (the $3-2$ spectra were smoothed to the angular resolution of the $2-1$ profiles), we estimate the 


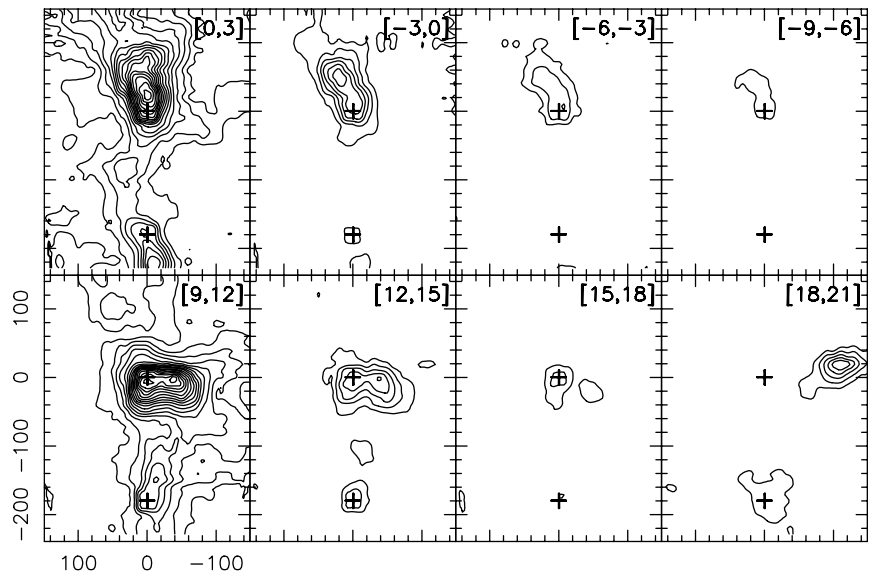

Fig. 6. Velocity channel map of $\mathrm{CO} J=3-2$. Each plot consists of the intensity integrated over a $3.0 \mathrm{~km} \mathrm{~s}^{-1}$ range, which is labeled at the right top corner of each plot. The lowest contour and the contour spacing are $1.0 \mathrm{~K} \mathrm{~km} \mathrm{~s}^{-1}$. The positions of I 20231 and $\left(0^{\prime \prime},-180^{\prime \prime}\right)$ are labeled as "+".

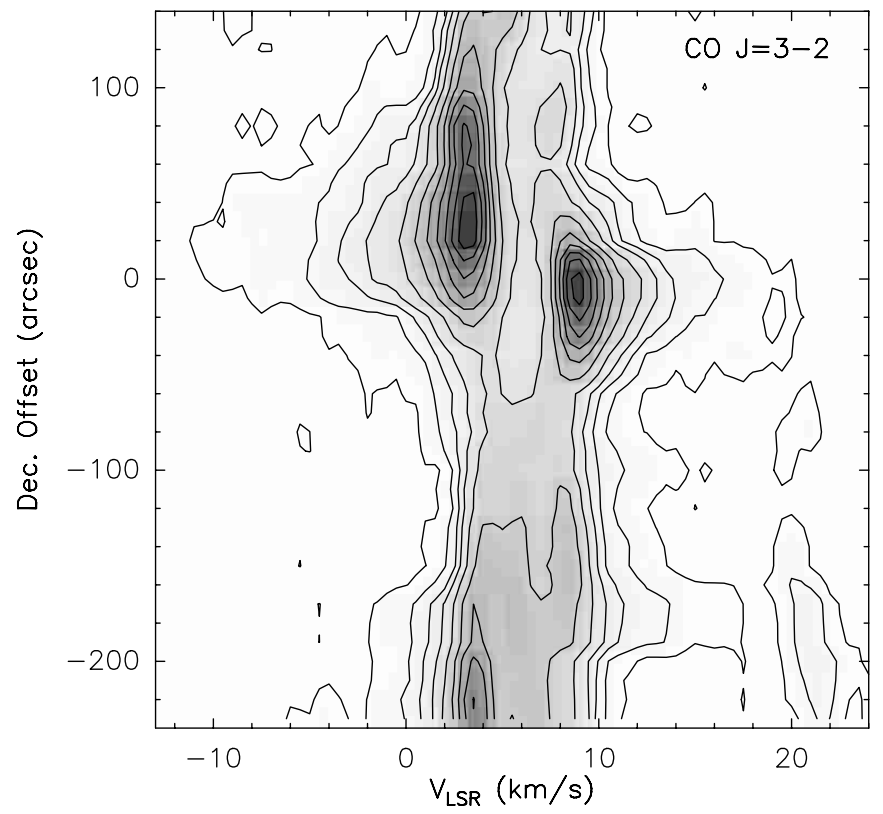

Fig. 7. Position-velocity map of $\mathrm{CO} J=3-2$ emission along Dec. for an RA offset $=0$. The contours and intervals are 0.3 to 2.1 by $0.6 \mathrm{~K}$, and 3 to 9 by $1 \mathrm{~K}$.

LTE excitation temperature $T_{\text {ex }}$ of the outflowing gas to be $20 \mathrm{~K}$ to $40 \mathrm{~K}$ for an extended source and $10 \mathrm{~K}$ to $14 \mathrm{~K}$ for a point source. We therefore take $T_{\mathrm{ex}}=20 \mathrm{~K}$ for our outflow parameter calculations described below.

Assuming that the $\mathrm{CO}$ emission is near LTE and that the ${ }^{12} \mathrm{CO}$ fractional abundance is $\left[{ }^{12} \mathrm{CO}\right] /\left[\mathrm{H}_{2}\right] \sim 10^{-4}$, the outflow parameters were estimated following a method discussed by Cabrit \& Bertout (1986, 1990). The mass of the outflowing gas is estimated from the integrated CO $J=3-2$ intensities of the red- and blueshifted lobes. The momentum $P$ and the energy $E$ are given by $M_{\text {flow }}<V>$ and $M_{\text {flow }}<V>^{2} / 2$, respectively, where $<V>$ is the intensity-weighted velocity. We can also get the entrainment mass rate $\dot{M}=M_{\text {flow }} / t_{\mathrm{d}}$, the force

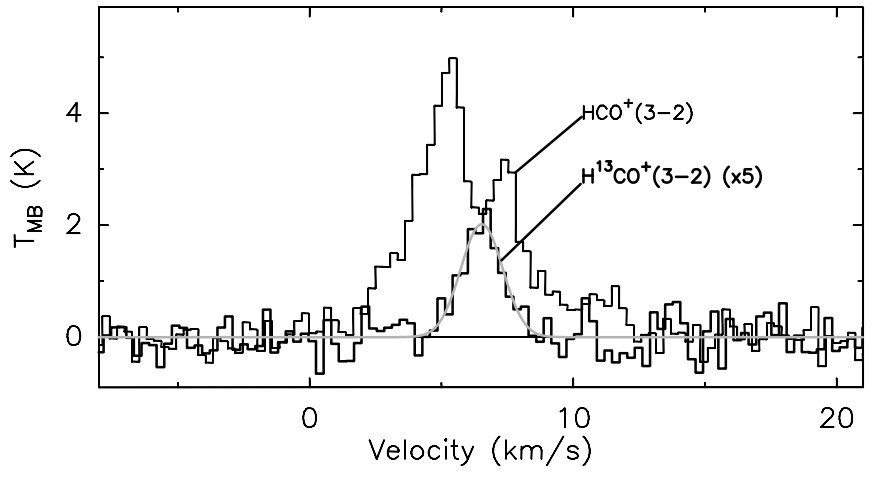

Fig. 8. $J=3-2$ spectra of $\mathrm{HCO}^{+}$(dark line) and $\mathrm{H}^{13} \mathrm{CO}^{+}(\times 5)$ (light line) taken at I 20231. The channel spacing is $0.28 \mathrm{~km} \mathrm{~s}^{-1}$.

in the flow $F=P / t_{\mathrm{d}}$, and the mechanical luminosity $L_{\text {mech }}=E / t_{\mathrm{d}}$, by taking the characteristic flow time scale, $t_{\mathrm{d}}=R_{\text {flow }} /<V>$, where the flow radius, $R_{\text {flow }}$ is the lobe extension. Correcting the flow parameters for an inclination angle (to the line of sight) of $45^{\circ}$, we summarize the outflow parameters in Table 2 . The corresponding parameters of the southern outflow are estimated assuming optically thin wing emission, since ${ }^{13} \mathrm{CO}$ data are not available in this case.

The parameters given in Table 2 are typical for lowmass or intermediate-mass YSOs. Cabrit \& Bertout (1990) estimated typical errors that should approximately be within a factor of $\sim 3$ for $M_{\text {flow }}$, a factor of $\sim 10$ for $F$, and a factor of $\sim 30$ for $L_{\text {mech }}$. These errors may be introduced by the uncertainties in $\left[{ }^{12} \mathrm{CO}\right] /\left[\mathrm{H}_{2}\right]$, the distance, line excitation temperature, inclination of the flow axis to the line of sight, and optical depth of the CO line. By using intensity weighted velocities rather than the maximum velocities, the values of $t_{\mathrm{d}}$ given in Table 2 may be overestimated. The flow radii $R_{\text {flow }}$ were corrected for an inclination of $45^{\circ}$ which is likely an overestimate for the northern outflow, but an underestimate for the southern outflow when we compare our observed line profiles, outflow structure and position-velocity maps to those of Cabrit \& Bertout (1990). In the case of the northern outflow, effects caused by taking "average" velocities and a "standard" inclination may cancel each other with respect to $t_{\mathrm{d}}$. In the case of the southern outflow, however, they add up so that the $t_{\mathrm{d}}$ value is an upper limit for the southern outflow. We can therefore safely conclude that the dynamical time of the northern flow is longer than that of the southern flow.

\section{3. $\mathrm{HCO}^{+}$and $\mathrm{H}^{13} \mathrm{CO}^{+}$}

The $\mathrm{HCO}^{+}$and $\mathrm{H}^{13} \mathrm{CO}^{+} J=3-2$ emission lines were measured in the central area of the northern core (see panels c) and d) in Fig. 4). Figure 8 shows the spectra taken towards I 20231. Strong $\mathrm{HCO}^{+}$self-absorption is observed at the peak velocity of the $\mathrm{H}^{13} \mathrm{CO}^{+}$line $\left(\sim 6.5 \mathrm{~km} \mathrm{~s}^{-1}\right)$. Weak blue- and red-shifted line wing emission is seen in the $J=3-2$ line of $\mathrm{HCO}^{+}$that can be interpreted as additional evidence for outflowing gas. The asymmetry in 
Table 2. Physical parameters of the two CO outflows ${ }^{a}$.

\begin{tabular}{c|c|ccccccccc}
\hline \hline \multicolumn{2}{c}{ Characteristic } & $\begin{array}{c}R_{\text {flow }} \\
(\mathrm{pc})\end{array}$ & $\begin{array}{c}<V> \\
\left(\mathrm{km} \mathrm{s}^{-1}\right)\end{array}$ & $\begin{array}{c}t_{\mathrm{d}} \\
\left(10^{4} \mathrm{yr}\right)\end{array}$ & $\begin{array}{c}M_{\text {flow }} \\
\left(M_{\odot}\right)\end{array}$ & $\begin{array}{c}\dot{M}^{b} \\
\left(M_{\odot} \mathrm{yr}^{-1}\right)\end{array}$ & $\begin{array}{c}P \\
\left(M_{\odot} \mathrm{km} \mathrm{s}^{-1}\right)\end{array}$ & $\begin{array}{c}F \\
\left(M_{\odot} \mathrm{km} \mathrm{s}^{-1} \mathrm{yr}^{-1}\right)\end{array}$ & $\begin{array}{c}E \\
\left(10^{45} \mathrm{ergs}^{2}\right.\end{array}$ & $\begin{array}{c}L_{\text {mech }} \\
\left(L_{\odot}\right)\end{array}$ \\
\hline \hline \multirow{3}{*}{ North } & Blue & 0.6 & 10.0 & 6.0 & 1.3 & $2.2 \times 10^{-5}$ & 12.7 & $2.1 \times 10^{-4}$ & 1.3 \\
& Red & 0.4 & 7.8 & 5.1 & 0.8 & $1.6 \times 10^{-5}$ & 6.0 & $1.2 \times 10^{-4}$ & 0.24 \\
& Total & $0.5^{c}$ & $8.9^{c}$ & $5.6^{c}$ & 2.1 & $3.8 \times 10^{-5}$ & 18.7 & $3.3 \times 10^{-4}$ & 0.10 \\
\hline \multirow{5}{*}{ South } & Blue & $0.3^{d}$ & 7.2 & 4.0 & 0.07 & $1.8 \times 10^{-6}$ & 0.50 & $1.3 \times 10^{-5}$ & 0.04 \\
& Red & 0.3 & 7.2 & 4.0 & 0.08 & $2.0 \times 10^{-6}$ & 0.58 & $1.4 \times 10^{-5}$ & 0.04 \\
& Total & $0.3^{c}$ & $7.2^{c}$ & $4.0^{c}$ & 0.15 & $3.8 \times 10^{-6}$ & 1.08 & $2.7 \times 10^{-5}$ & 0.007 \\
\hline
\end{tabular}

${ }^{a}$ An inclination angle of $45^{\circ}$ has been taken into account for all parameters listed in this table. ${ }^{b}$ Note the $\dot{M}$ here is the entrainment rate. ${ }^{c}$ The average of two lobes. ${ }^{d}$ Due to lack of coverage in the southern outflow, $R_{\text {flow }}$ for the blueshifted lobe is assumed to be equal to that of its redshifted counterpart.

the line showing enhanced blue-shifted emission characterizes all spectra within a radius of $\sim 40^{\prime \prime}(\sim 0.2 \mathrm{pc})$ around I 20231. This is indicative of possible infall motion that will be discussed in Sect. 6.4.

The total integrated intensity map of $\mathrm{H}^{13} \mathrm{CO}^{+}$shows a very compact core with a deconvolved half-power size of about $20^{\prime \prime}$ by $35^{\prime \prime}$ ( 0.1 pc by $0.17 \mathrm{pc}$; see panel $\mathrm{d}$ in Fig. 4). With the same method used above, we estimate a fractional $\mathrm{HCO}^{+}$abundance of $9.6 \times 10^{-10}$, about an order of magnitude lower than the typical value of $\sim 8 \times 10^{-9}$ in dark clouds (Irvine et al. 1987), but close to the inferred value of $1 \times 10^{-9}$ for envelopes of submillimeter continuum sources in the Serpens molecular cloud (Hogerheijde et al. 1999). This may indicate a depletion of $\mathrm{HCO}^{+}$in this region, i.e. some molecules are frozen out onto the dust. The mass associated with $\mathrm{H}^{13} \mathrm{CO}^{+}$is $\sim 50 M_{\odot}$, consistent with the mass derived from $\mathrm{C}^{17} \mathrm{O}$.

As already mentioned, broad-line wings were detected in the $\mathrm{HCO}^{+} J=3-2$ spectra. The outflowing emission shown in Fig. 9 was integrated over a velocity range of 3 to $5 \mathrm{~km} \mathrm{~s}^{-1}$ for blueshifted (solid contours) emission and 7.5 to $9 \mathrm{~km} \mathrm{~s}^{-1}$ for redshifted (dashed contours) emission, respectively. By assuming LTE at an excitation temperature $T_{\mathrm{ex}}=20 \mathrm{~K}$ and optically thin outflow emission, we estimate the total mass of outflowing gas traced by $\mathrm{HCO}^{+}$in the velocity range mentioned above to be $6 M_{\odot}$, almost three times the gas flow traced by CO. This is mostly due to the inclusion of the low velocity outflowing gas which could not directly be identified in the broader line profiles of $\mathrm{CO}$. We thus find a total mass of $8 M_{\odot}$ for the outflow. Although such a mass estimate depends on uncertain molecular abundances, we can conclude that a significant amount of the outflowing gas is observed at low velocities and that the total outflowing gas mass is severely underestimated by the $\mathrm{CO}$ data alone.

\section{4. $C S$ and $C^{34} S$}

In contrast to $\mathrm{CO}$ and $\mathrm{HCO}^{+}$our $\mathrm{CS}$ profiles show no sign of self-absorption. With a critical density of about $7 \times 10^{6} \mathrm{~cm}^{-3}$, the $J=5-4$ transition of CS and its isotopes traces the very dense molecular gas. While CS and

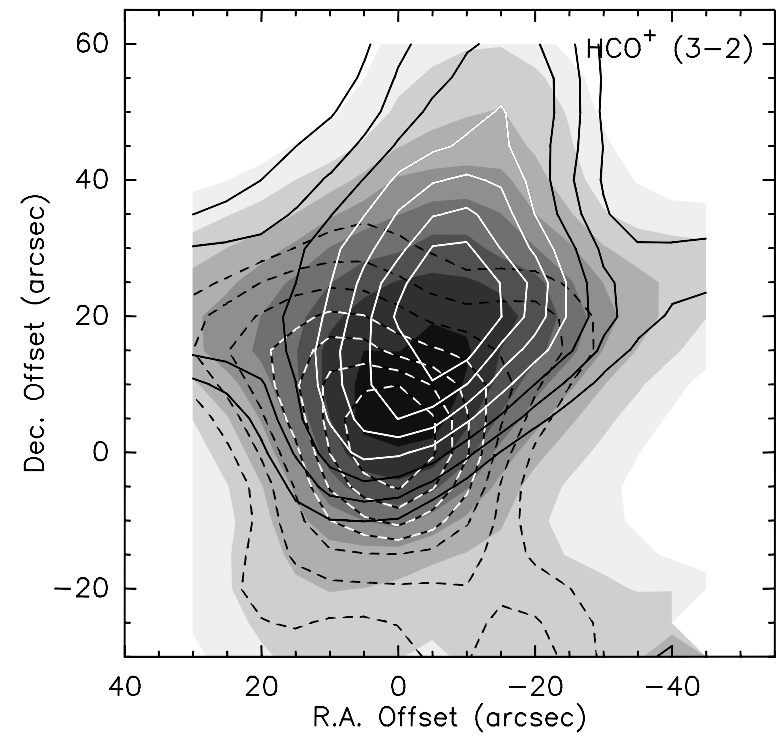

Fig. 9. Bipolar outflow (contours) overlaid on the total integrated intensity map (grey) of $\mathrm{HCO}^{+} J=3-2$ taken around I 20231. The blue- (solid) and the redshifted (dashed) emission is integrated from 3 to $5 \mathrm{~km} \mathrm{~s}^{-1}$ and 7.5 to $9 \mathrm{~km} \mathrm{~s}^{-1}$, respectively. Contour levels are $30 \%, 40 \%, \ldots, 90 \%$ of the peak values, which are 5.7 and $7.2 \mathrm{~K} \mathrm{~km} \mathrm{~s}^{-1}$, respectively. Grey scales are 4.4 to 19.8 by steps of $2.2 \mathrm{~K} \mathrm{~km} \mathrm{~s}^{-1}$.

$\mathrm{C}^{34} \mathrm{~S}$ were detected towards I $20231, \mathrm{C}^{33} \mathrm{~S}$ and ${ }^{13} \mathrm{CS}$ remain undetected with an rms level of $\sim 20 \mathrm{mK}$ and a channel separation of $0.3 \mathrm{~km} \mathrm{~s}^{-1}$. A Gaussian fit to the CS $J=5-4$ profile at the $\left(0^{\prime \prime}, 0^{\prime \prime}\right)$ position gives a velocity of $6.3 \mathrm{~km} \mathrm{~s}^{-1}$, the same as that for the $\mathrm{C}^{18} \mathrm{O} J=2-1$ line but different from that of CS $J=2-1$ (Bronfman et al. 1996). We believe that this is mainly due to different optical depths, i.e., optically thick emission in the $J=2-1$ line and optically thin emission in the $5-4$ line. A nine point CS map was also taken towards the southern core with the peak located at $\left(0^{\prime \prime},-180^{\prime \prime}\right)$. As shown in Table 1 , the CS emission lines are weaker and narrower towards the southern peak, and the emission appears to be much more compact than that in $\mathrm{NH}_{3}$. The integrated CS intensity map (Fig. 4, panel e) shows a slightly elongated structure in N-S direction with a peak about $10^{\prime \prime}$ north of I 20231. 


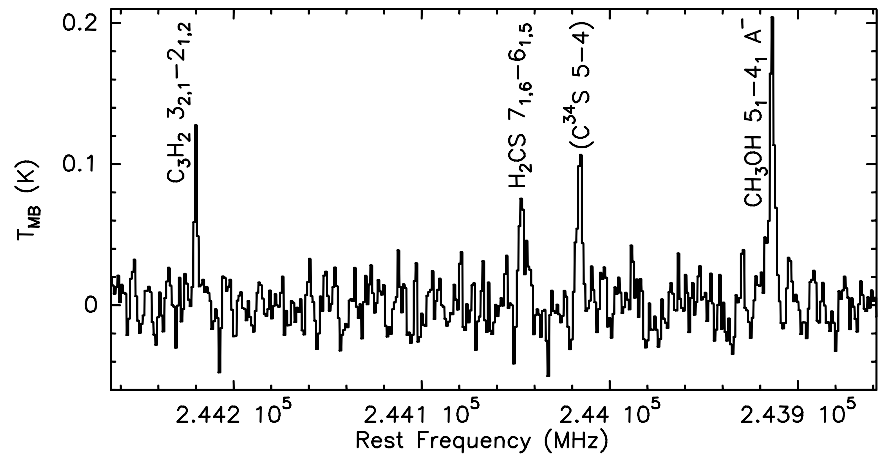

Fig. 10. Dual sideband spectrum with the $\mathrm{C}^{34} \mathrm{~S} J=5-4$ line from the signal sideband and the other lines from the image sideband. Identified molecular transitions are marked. The channel spacing is $0.62 \mathrm{~km} \mathrm{~s}^{-1}$.

Assuming the same excitation temperature for the $J=5-4$ lines of $\mathrm{CS}$ and $\mathrm{C}^{34} \mathrm{~S}$, we can estimate the CS optical depth for a given isotopic abundance ratio $R$ (e.g. Zinchenko et al. 1994). With $R=31.3$ for I 20231 (Chin et al. 1996), the excitation temperature, the peak optical depth and the mean optical depth averaged over the line profile are estimated to be $6.5 \mathrm{~K}, 2.2$ and 1.8 . We then derive a CS fractional abundance of $7.8 \times 10^{-9}$, and a core mass of about $26 M_{\odot}$.

While $\mathrm{C}^{34} \mathrm{~S} J=5-4$ was detected in the lower sideband, $\mathrm{CH}_{3} \mathrm{OH} 5(1)-4(1) \mathrm{A}^{-}(243.9158 \mathrm{GHz}), \mathrm{H}_{2} \mathrm{CS}_{1,6^{-}}$ $6_{1,5}(244.0478 \mathrm{GHz})$ and $\mathrm{C}_{3} \mathrm{H}_{2} 3_{2,1}-2_{1,2}(244.2221 \mathrm{GHz})$ were detected in the upper sideband with peak intensities of $0.19,0.10$ and $0.14 \mathrm{~K}$ and integrated intensities of 0.63 , 0.57 , and $0.27 \mathrm{~K} \mathrm{~km} \mathrm{~s}^{-1}$, respectively (see Fig. 10). Since these lines are all from non-linear molecules, Eq. (1) cannot be used to estimate the column densities. Instead, we have used a relationship between the energy of the upper level and the integrated line intensity (see e.g. Groesbeck et al. 1994) for an optically thin transition, adopting the line strength and partition function information from the JPL line catalog (Pickett et al. 1998 and online at http://spec.jpl.nasa.gov). Under the assumption of LTE and optically thin line emission, taking $T_{\mathrm{ex}}=10 \mathrm{~K}$, we estimate $\mathrm{CH}_{3} \mathrm{OH}, \mathrm{H}_{2} \mathrm{CS}$ and $\mathrm{C}_{3} \mathrm{H}_{2}$ column densities to be $6.6 \times 10^{14}, 9.0 \times 10^{13}$ and $1.1 \times 10^{13} \mathrm{~cm}^{-2}$, and thus fractional abundances to be $9.7 \times 10^{-9}, 1.3 \times 10^{-9}$ and $1.6 \times 10^{-10}$, respectively. These abundances are lower limits. If the lines are not optically thin, column densities and abundances would be higher. Note that abundances are estimated assuming that molecular lines are sampling the same volume as the $870 \mu \mathrm{m}$ dust continuum emission (see Sect. 3). This does not necessarily hold, since different molecular species and even transitions of the same species have fairly different critical densities. Multi-line observations and radiative transfer modeling are needed to test this hypothesis for I 20231.

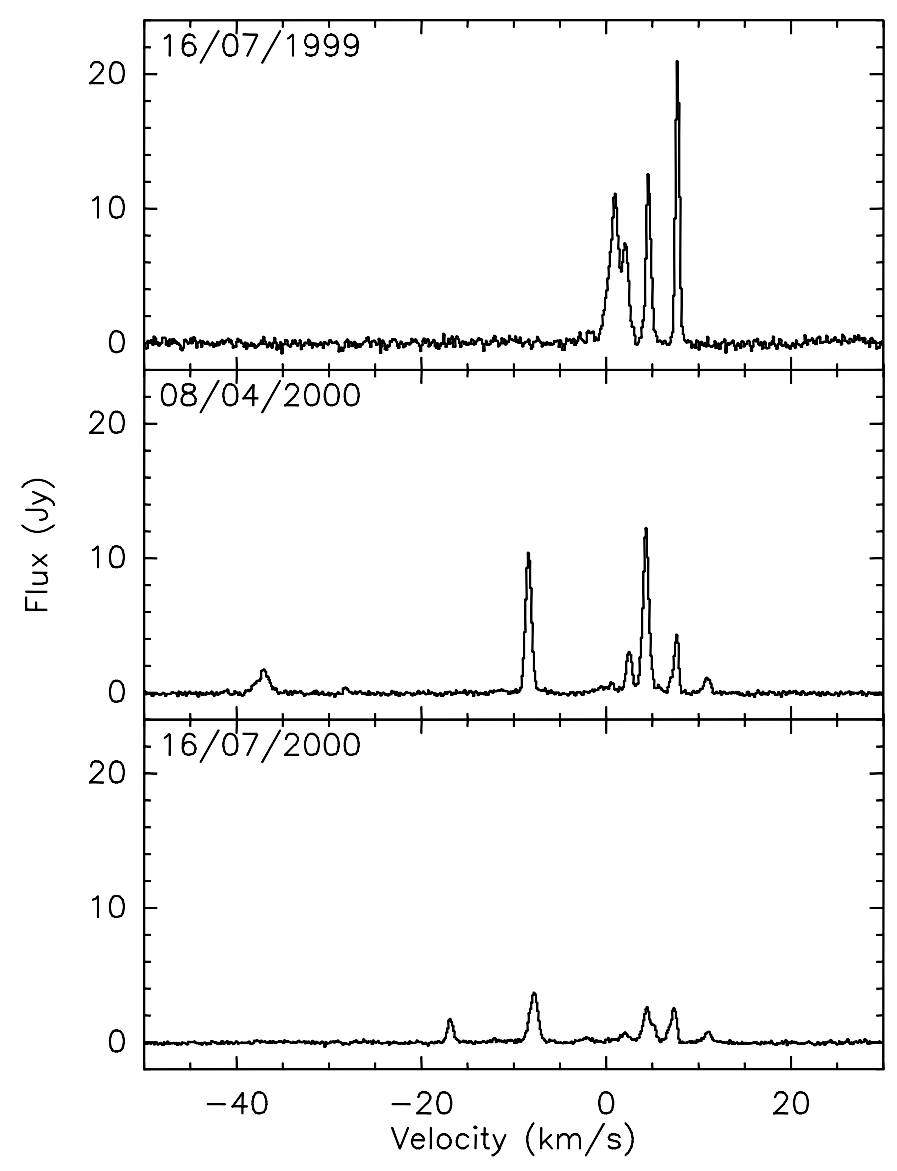

Fig. 11. Three $22 \mathrm{GHz} \mathrm{H}_{2} \mathrm{O}$ maser spectra taken at I 20231 with the epoch labeled at the left top corner of each panel. The channel spacing is $0.13 \mathrm{~km} \mathrm{~s}^{-1}$. The full observed velocity range is $\sim-110$ to $140 \mathrm{~km} \mathrm{~s}^{-1}$. Only those parts of the spectra are shown that contain detected emission.

\subsection{Water maser emission}

Figure 11 shows $22 \mathrm{GHz}$ water maser spectra taken with the Effelsberg 100-m telescope. As shown in the spectra, the velocity ranges of maser emission are between $-40 \mathrm{kms}^{-1}$ and $+12 \mathrm{kms}^{-1}$, with more blue features than red ones relative to the systemic velocity of $6.3 \mathrm{~km} \mathrm{~s}^{-1}$. The maser spectra also show time variability on a timescale $\leq 3$ months. While the total $\mathrm{H}_{2} \mathrm{O}$ maser luminosity decreased by a factor of $\sim 3$ from $40.8 \mathrm{Jy} \mathrm{km} \mathrm{s}^{-1}$ in July 1999 to $14.3 \mathrm{Jy} \mathrm{km} \mathrm{s}^{-1}$ in July 2000, some individual maser components showed even stronger variations. Combining our data with the water maser spectra reported by Brand et al. (1994), the total $\mathrm{H}_{2} \mathrm{O}$ luminosity $L_{\mathrm{H}_{2} \mathrm{O}}$, assuming isotropic emission, varied between $3.3 \times 10^{-7}$ and $1.1 \times 10^{-6} L_{\odot}$. The upper limit is consistent with what one expects for a maser associated with an IRAS source with $L_{\mathrm{FIR}} \sim 10^{2} L_{\odot}$ (Henning et al. 1992). From the mapped maser emission in April 2000, almost all the maser components, e.g. those at $-37.1,-8.26$, $4.33,7.59$ and $11.00 \mathrm{~km} \mathrm{~s}^{-1}$, are centered at the IRAS position within a pointing accuracy of $\sim 5^{\prime \prime}$, except the $2.48 \mathrm{~km} \mathrm{~s}^{-1}$ feature which peaked at $\left(0^{\prime \prime}, 10^{\prime \prime}\right)$. We also observed the $\left(0^{\prime \prime},-180^{\prime \prime}\right)$ position (i.e. the southern $\mathrm{NH}_{3}$ and 


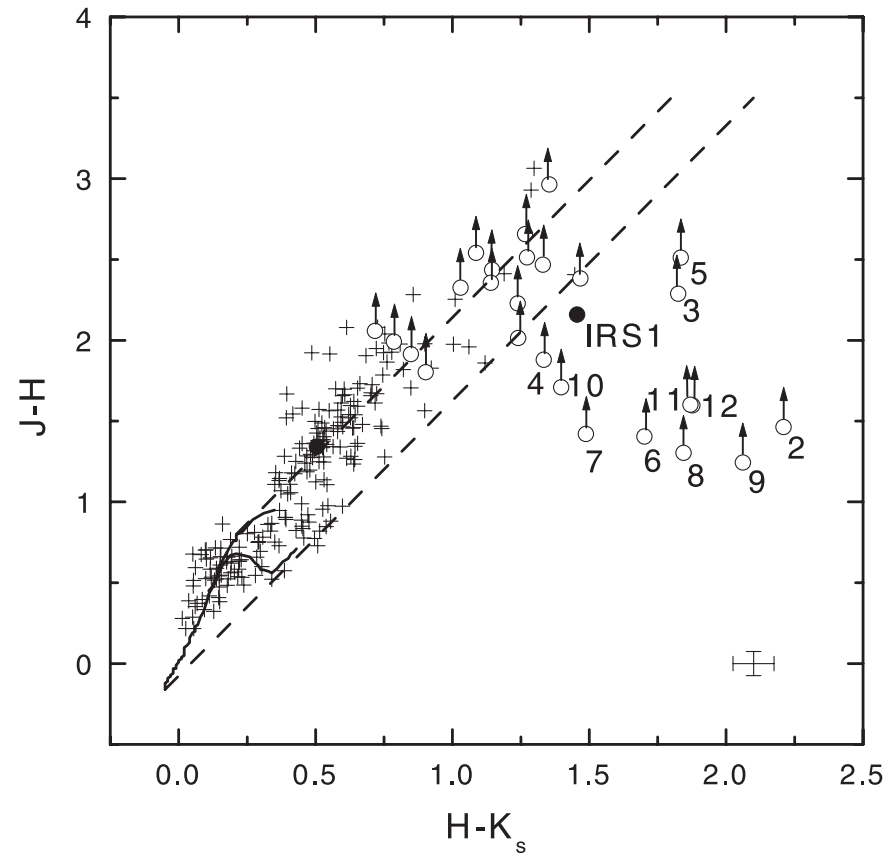

Fig. 12. Color-color diagram of the sources extracted from the 2MASS data. The circles represent YSOs. The solid curves show the locus of the unreddened main sequence and giant stars, the two parallel dashed lines form the reddening band and confine the region in which stars with normal photosphere fall. A solid circle marks the positions of star IRS1 (=I 20231). Sources not detected in $J$ band are shown as open circles. Arrows indicate the lower limits, for which the $J$ band completeness limit of $17 \mathrm{mag}$ is adopted for $J$ magnitudes. YSO candidates are labeled with sequence number from 2 and 12 according to their offset from I 20231. In the lower right corner the average photometric error cross is indicated.

sub-mm continuum peak), but no signal was seen at an rms noise level of $0.1 \mathrm{Jy}$ (channnel spacing: $0.13 \mathrm{~km} \mathrm{~s}^{-1}$ ), which yields an upper limit of $L_{\mathrm{H}_{2} \mathrm{O}} \sim 3 \times 10^{-10} L_{\odot}$.

\section{2MASS NIR photometry}

Stars within $8^{\prime}$ diameter of I 20231 are extracted from The Two Micron All Sky Survey (2MASS, see http://www.ipac. caltech. edu/2mass/ for details) catalogue which contains $J, H$ and $K_{\mathrm{s}}$ magnitudes of stars. Completeness limits in magnitude are close to 17,16 and $15 \mathrm{mag}$ in the $J, H$ and $K_{\mathrm{s}}$ bands, respectively. The $J-H / H-K_{\mathrm{s}}$ color-color diagram presented in Fig. 12 includes stars detected at least in $H$ and $K_{\mathrm{s}}$ with magnitudes of $6.0 \leq m\left(K_{\mathrm{s}}\right) \leq 14.3$ and photometric uncertainties less than $0.2 \mathrm{mag}$ in all detected bands. Sources satisfying the above criteria but not being detected in $J$ band are denoted with open circles. The completeness limit of $17 \mathrm{mag}$ is adopted as a lower limit of $J$ magnitudes for these sources. While the solid curve draws the locus of points corresponding to unreddened main sequence and giant branch stars (Koornneef 1983), the two parallel dashed lines form the reddening band and are bound to the region of stars with normal photospheres
Table 3. 2MASS NIR properties of embedded sources.

\begin{tabular}{rccccc}
\hline \hline No. & $\begin{array}{c}\text { RA(2000) } \\
(\mathrm{h} \text { m s })\end{array}$ & $\begin{array}{c}\text { Dec. }(2000) \\
\left(\circ \quad{ }^{\circ} \quad\right.\end{array}$ & $\begin{array}{c}J \\
(\mathrm{mag})\end{array}$ & $\begin{array}{c}H \\
(\mathrm{mag})\end{array}$ & $\begin{array}{c}K_{\mathrm{s}} \\
(\mathrm{mag})\end{array}$ \\
\hline IRS 1 & 202507.2 & +345005 & 13.37 & 11.21 & 9.76 \\
2 & 202507.3 & +344956 & - & 15.54 & 13.33 \\
3 & 202504.8 & +345014 & - & 14.71 & 12.89 \\
4 & 202507.0 & +344931 & - & 15.12 & 13.79 \\
5 & 202504.3 & +345008 & - & 14.49 & 12.66 \\
6 & 202506.6 & +344915 & - & 15.60 & 13.90 \\
7 & 202507.4 & +345123 & - & 15.58 & 14.09 \\
8 & 202503.4 & +345120 & - & 15.70 & 13.85 \\
9 & 202458.3 & +344939 & - & 15.76 & 13.70 \\
10 & 202505.2 & +345213 & - & 15.29 & 13.89 \\
11 & 202505.6 & +344736 & - & 15.54 & 13.88 \\
12 & 202502.9 & +344613 & - & 15.40 & 13.53 \\
\hline
\end{tabular}

(see Lada \& Adams 1992). The sources lying to the right side of the reddening band are believed to be mostly YSOs (Lada \& Adams 1992; Lada et al. 1993). Careful scrutiny of the region reveals 12 YSO candidates (represented by circles labeled with sequence numbers in Fig. 12, see also Fig. 1). Since almost all these YSOs except for IRS1 (solid circle) were not detected in $J$, their positions in the color-color diagram are still uncertain. Deep photometric observations are therefore needed to confirm their YSO nature. Table 3 includes their near-infrared photometric data. From its location on the color-color diagram, we can estimate the extinction of IRS1 to be $\sim 15$, much lower than that derived from the peak emission of the $870 \mu \mathrm{m}$ dust continuum $\left(A_{\mathrm{V}} \sim 100\right)$. Although the $A_{\mathrm{V}}$ estimate using dust emission is model dependent (see Sect. 3), the difference in the extinction estimates is most likely beyond the uncertainties induced by dust properties. This may suggest that the core is structured and/or that IRS1 is not at its center, in which case SMM1 and IRS1 may not refer to the same source. This is further supported by the fact that IRS1 is located $\sim 8^{\prime \prime}(\sim 0.04 \mathrm{pc})$ northwest of the $870 \mu \mathrm{m}$ peak (SMM1; see also Sect. 6.2).

\section{Discussion}

\subsection{Physical properties of the two cores}

Dense cores are the birthplaces of stars. Their study provides deep insights into the initial conditions of cool dense gas clouds that determine important stellar parameters like mass, metallicity, and initial rotation period. Jijina et al. (1999) compiled a catalog of 264 dense cores from $\mathrm{NH}_{3}$ observations, and found that the presence of cluster associations has a dramatic effect on core gas and YSO properties. The line widths, kinetic temperatures and core sizes for cores in clusters are much larger than the corresponding physical parameters for cores in isolated starforming environments. 
The intrinsic line widths averaged over the $\mathrm{NH}_{3}$ cores are 1.79 and $1.20 \mathrm{~km} \mathrm{~s}^{-1}$ for the northern and the southern source, respectively. These values are very close to the cluster associated median values of $1.20 \mathrm{~km} \mathrm{~s}^{-1}$ from the Jijina samples (samples IV and V in Jijina 1999) and larger than the median values for those samples without association (samples I, II and III in Jijina et al. 1999). Moreover, turbulence in both cores is predominanantly non-thermal, since for the molecule of mean mass $\left(=1.36 \mathrm{~m}_{\mathrm{H}_{2}}\right)$ thermal line widths $\Delta v_{\mathrm{T}}$ (0.63 and $0.49 \mathrm{~km} \mathrm{~s}^{-1}$ for the northern and the southern core, respectively) determined from the kinetic temperature are small relative to the nonthermal line widths $\Delta v_{\mathrm{NT}}(1.77$ and $1.18 \mathrm{~km} \mathrm{~s}^{-1}$ ), obtained by subtracting in quadrature the thermal line width from the intrinsic line width averaged over the core. Further supported by substantial core masses, the two $\mathrm{NH}_{3}$ cores are reminiscent of the "turbulent, massive dense cores" forming clusters or groups of stars in the Jijina et al. samples, although none of the two cores seems to have more than 30 embedded stars associated. This is supported by the distribution of the YSO candidates shown in Fig. 1, in which at least six (IRS1, 2 to 6) appear to be associated with the northern core and another two with the southern one (11 and 12).

Towards the northern core, there seems to be a temperature gradient from the core center to the outermost layer. The detection of the $(4,4)$ line of $\mathrm{NH}_{3}$ yields a kinetic temperature of $\sim 30 \mathrm{~K}$ at the position of I 20231 . This value is an average over the beam size of $40^{\prime \prime}(\sim 0.2 \mathrm{pc})$ and is likely a lower limit for the region immediately surrounding I 20231, since the $(4,4)$ line was only detected towards the IRAS position and may not have been spatially resolved. The average kinetic temperature over the rest of the core is $\sim 15 \mathrm{~K}$. For regions where $\mathrm{NH}_{3}$ is detectable, the density is on the order of $10^{4} \mathrm{~cm}^{-3}$. At lower densities $\left(10^{2-3} \mathrm{~cm}^{-3}\right)$, an excitation temperature of $\sim 11 \mathrm{~K}$ is traced by $\mathrm{C}^{18} \mathrm{O}$, decreasing further to $\sim 7.5 \mathrm{~K}$ towards the outermost layer of the clouds (see Sect. 4.2.1).

\subsection{The driving sources of the outflows}

The far-infrared luminosity of the IRAS source, calculated from the IRAS flux, is $L_{\mathrm{FIR}}=196 L_{\odot}$ (at a distance of $1 \mathrm{kpc}$ ). This luminosity is higher than the minimum value set by the mechanical luminosity requirement. By adopting the $\dot{M}-L_{\text {bol }}$ relationship of Shepherd \& Churchwell (1996), the luminosity of the outflow source in I 20231 is predicted to be greater than $114 L_{\odot}$. Within the error range, it suggests that the infrared source in question can provide sufficient mechanical power to drive the $\mathrm{CO}$ outflow.

Source IRS1, located at $\left(3^{\prime \prime}, 0^{\prime \prime}\right)$, coincides well with the IRAS source I 20231 with $K_{\mathrm{s}}=9.76 \mathrm{mag}$ and $H-K_{\mathrm{s}}=$ 1.46 mag. Immediately next to IRS1, a fainter $\left(K_{\mathrm{s}}=\right.$ $13.33 \mathrm{mag})$ companion source 2 was found at $\left(4^{\prime \prime},-11^{\prime \prime}\right)$, appearing much redder with $H-K_{\mathrm{s}}=2.21 \mathrm{mag}$. Are they physically related? And which one is responsible for the outflow? Considering the clumpiness in outflow structure, are perhaps both stars driving the outflow? It is worth noting that the position difference between source 2 and SMM1 is $5^{\prime \prime}$, which is marginally within the pointing uncertainty of the $870 \mu \mathrm{m}$ observations. The question then arises on how IRS1, source 2 and SMM1 are physically related. Considering the relatively large beam $\left(22^{\prime \prime}\right)$ of the $870 \mu \mathrm{m}$ observations, higher resolution dust continuum and molecular line observations as well as some deeper NIR images are needed to answer these questions.

In the southern core, we did not find such YSO candidates close to SMM2. Although there is a much weaker (14.13 mag) $K_{\mathrm{s}}$ source located at $\left(-3^{\prime \prime},-180^{\prime \prime}\right)$, its nature is unknow since it was not detected in $J$ and $H$ and we cannot place it on the color-color diagram. Most likely the driving source of the southern outflow is still deeply embedded in the cloud. It is also less luminous than its counterpart(s) of the northern outflow.

\subsection{Evolutionary status of the two cores}

Protostars evolve from Class 0 to Class I, II, III. Class 0 sources, the youngest protostars, are characterized by $M_{\text {env }} / L_{\text {bol }}>0.1 M_{\odot} / L_{\odot}\left(M_{\text {env }}\right.$ is the envelope mass $)$, no detectable emission at wavelengths shorter than $\sim 10 \mu \mathrm{m}$, and the presence of outflow (André et al. 1993, 2000). At present, 42 Class 0 sources have been identified (André et al. 2000).

A reasonably good estimate of $M_{\text {env }}$ can be provided by the strength of the optically thin $870 \mu \mathrm{m}$ continuum emission in the $22^{\prime \prime}$ beam $(\sim 0.1 \mathrm{pc}$, the typical size of protostellar envelopes in the Taurus cloud). Following the method used in Sect. 3, we can estimate $M_{\text {env }}$ for SMM1 and SMM2 to be about 9 and $14 M_{\odot}$, with their peak flux densities of 1.67 and $0.60 \mathrm{Jy}^{-1}$ beam $^{-1}$, respectively. It is, however, worth noting that $\beta=1.5\left(\kappa_{870 \mu \mathrm{m}}=\right.$ $0.02 \mathrm{~cm}^{2} \mathrm{~g}^{-1}$ correspondingly), a value lower than the one used for mass calculations in Sect. 3, is found to be more suitable to estimate the masses traced by the dust around protostars (e.g. Visser et al. 1998, 2001). Moreover, the averaged dust temperature within a $22^{\prime \prime}$ beam could be higher than the ones used in Sect. 3. Both effects lead to lower masses. Considering additionally that I 20231 may not be at the core center, we can safely conclude that $M_{\mathrm{env}} / L_{\mathrm{bol}}<0.04 M_{\odot} / L_{\odot}$ for SMM1, typical of a Class I source. The detection of I 20231 at $12 \mu \mathrm{m}$ and $2 \mu \mathrm{m}$ and the moderate strength of its $\mathrm{CO}$ outflow also suggest SMM1 to be a $\sim 200 L_{\odot}$ Class I YSO.

To better constrain the properties of SMM2, we first estimate the lower limit to $M_{\text {env }} . \kappa_{870 \mu \mathrm{m}}=0.03 \mathrm{~cm}^{2} \mathrm{~g}^{-1}$ is most likely an upper limit if we take the assumption from Hildebrand (1983), i.e. $\kappa_{\lambda}=0.1(250 / \lambda(\mu \mathrm{m}))^{\beta} \mathrm{cm}^{2} \mathrm{~g}^{-1}$, with $\beta=1$, which is the lower limit in star forming regions at the millimeter and submillimeter wavelengths. A dust temperature of $30 \mathrm{~K}$ is most likely an upper limit to SMM2. These parameters yield a lower limit to the envelope mass of $1 M_{\odot}$. Meanwhile, since there is no 
detectable IRAS source associated with SMM2, the sensitivity of the IRAS instruments $(0.5 \mathrm{Jy}$ at 12,25 , and $60 \mu \mathrm{m}, 1.5 \mathrm{Jy}$ at $100 \mu \mathrm{m}$ ) allow us to estimate an upper limit of $L_{\mathrm{bol}} \sim 6 L_{\odot}$ for the luminosity of the possible source(s) embedded in the southern core. The $M_{\text {env }} / L_{\mathrm{bol}}$ ratio for SMM2 is therefore comfortably greater than $0.1 M_{\odot} / L_{\odot}$, typical of a Class 0 source. Combined with the fact that SMM2 also harbors a bipolar outflow, the southern core is more likely to contain a Class 0 object with $1 M_{\odot} \lesssim M_{\text {env }} \lesssim 14 M_{\odot}$.

Since outflows are found in both cores, we can also compare the evolutionary phases of the two cores by placing these two sources on the outflow efficiency versus $M_{\text {env }} / L_{\text {bol }}^{0.6}$ diagram (see Fig. 7 of Bontemps et al. 1996). The outflow efficiency is the outflow force divided by the radiative momentum flux $\left(L_{\mathrm{bol}} / c\right.$ ) and is $\sim 80$ (or $\sim 380$ also accounting for the low velocity flow traced by $\mathrm{HCO}^{+}$) for the northern outflow and $>220$ for the southern one. Accounting for the different $M_{\text {env }} / L_{\text {bol }}^{0.6}$ values of the two cores, the northern one lies in the transit region between Class 0 and I, while the southern one lies distinctly in the region occupied by Class 0 sources. This, in agreement with the fact that the dynamical time of the northern flow is longer than that of the southern flow (see Sect. 4.2.2), further supports the protostellar status of SMM1 (I 20231) and SMM2.

We thus conclude that the southern core is in an earlier evolutionary stage than the northern core. One may ask why there is no water maser detection in the southern core, since Class 0 sources are believed to be favorable sites for such masers (Furuya et al. 2001). An answer could be that the maser is too weak to be detected at an rms level of $0.1 \mathrm{Jy}$, since according to the $L_{\mathrm{H}_{2} \mathrm{O}}-L_{\mathrm{bol}}$ correlation found by Furuya et al. (2001), we expect for a protostar with $L_{\text {bol }}<6 L_{\odot}$ a luminosity of $L_{\mathrm{H}_{2} \mathrm{O}} \lesssim 1.7 \times 10^{-11} L_{\odot}$. This is about two orders of magnitude below the estimated upper limit (see Sect. 4.5). On the other hand, the nondetection of a $\mathrm{H}_{2} \mathrm{O}$ maser in the southern core is not inconsistent with the recent statistical study by Zhang et al. (2001) who found that the outflow may develop before the appearance of a water maser although their study is based on a sample of massive star forming regions. It is therefore also possible that the southern core has not yet reached an evolutionary stage to form a luminoous $\mathrm{H}_{2} \mathrm{O}$ maser, while an outflow was already activated.

The above discussion is based on the assumption that SMM1 and IRS1 refer to the same source as I 20231, but this is not necessarily the case. As we have mentioned in Sects. 5 and 6.2 , the differences in extinction $\left(A_{\mathrm{v}} \sim 100\right.$ versus $\sim 15)$ and in position between SMM1 and IRS1 $\left(\sim 8^{\prime \prime}\right.$ or $\left.0.04 \mathrm{pc}\right)$ raise the possibility that the two sources are distinct objects of the northern "cluster". In such a case, $L_{\mathrm{bol}}$ of SMM1 would be significantly lower than the value estimated from FIR flux densities of I 20231, and as a result SMM1 might still be a Class 0 object and driving source of the northern outflow. High angular resolution observations would be required to draw definite conclusions.
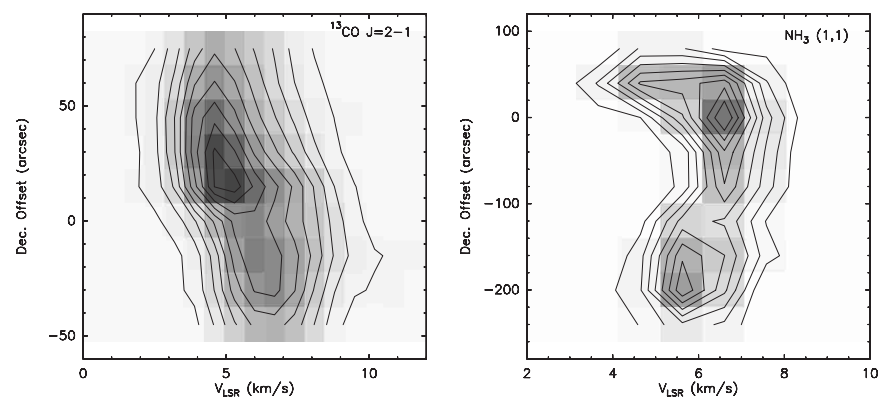

Fig. 13. Position-velocity maps of ${ }^{13} \mathrm{CO} J=2-1$ (left) and $\mathrm{NH}_{3}(1,1)$ (right) emission along Dec. for RA offset $=0$. The contours are $10 \%$ to $90 \%$ by $10 \%$ of the peak emission $(9.2 \mathrm{~K}$ and $4.1 \mathrm{~K}$ for ${ }^{13} \mathrm{CO} J=2-1$ and $\mathrm{NH}_{3}(1,1)$, respectively).

\subsection{Velocity gradients and the asymmetric line profiles}

There exists a velocity gradient from north to south in all molecular spectra, indicating an internal motion. In the case of the $\mathrm{NH}_{3}$ lines, the velocity increases monotonously from $5.2 \mathrm{~km} \mathrm{~s}^{-1}$ at $\left(0^{\prime \prime}, 80^{\prime \prime}\right)$ to $6.8 \mathrm{~km} \mathrm{~s}^{-1}$ at $\left(0^{\prime \prime},-80^{\prime \prime}\right)$, where there seems to be a turning point. Further south the velocity shifts back to and stays almost constant at $6.0 \mathrm{~km} \mathrm{~s}^{-1}$. Such a gradient is also found in ${ }^{13} \mathrm{CO} J=$ $2-1$, where the velocity shifts from $5 \mathrm{~km} \mathrm{~s}^{-1}$ at $\left(0^{\prime \prime}, 75^{\prime \prime}\right)$ to $6.3 \mathrm{~km} \mathrm{~s}^{-1}$ at $\left(0^{\prime \prime},-45^{\prime \prime}\right)$. The CS $J=5-4$ line profile at the central position is quite similar to that of the ${ }^{13} \mathrm{CO}$ line, with a peak slightly skewed to low velocity. Again, the velocity increase from $4.5 \mathrm{~km} \mathrm{~s}^{-1}$ at $\left(0^{\prime \prime}, 75^{\prime \prime}\right)$ to $6.5 \mathrm{~km} \mathrm{~s}^{-1}$ at $\left(0^{\prime \prime},-30^{\prime \prime}\right)$, and drops to $6.0 \mathrm{~km} \mathrm{~s}^{-1}$ further south just like ammonia. This indicates that a boundary exists near $\left(0^{\prime \prime},-100^{\prime \prime}\right)$, separating the northern cloud (with a "systemic" velocity $\sim 6.3 \mathrm{~km} \mathrm{~s}^{-1}$ ) from the southern cloud (with $\sim 6.0 \mathrm{~km} \mathrm{~s}^{-1}$ ). The gradients across the northern core are 2 to $4 \mathrm{~km} \mathrm{~s}^{-1} \mathrm{pc}^{-1}$, slightly larger than the typical rotation induced gradients of $1 \mathrm{~km} \mathrm{~s}^{-1} \mathrm{pc}^{-1}$ for molecular cloud cores (Goodman et al. 1993), but within the range found by Jijina et al. (1999) in their sample. As examples, we present in Fig. 13 the position-velocity maps of ${ }^{13} \mathrm{CO}$ $J=2-1$ (left) and $\mathrm{NH}_{3}(1,1)$ (right). Morphologically, the diagrams are consistent with a large cloud that shows overall large scale rotation. However, such an effect could also be caused by two independent cores with slightly different velocities.

The $\mathrm{HCO}^{+} J=3-2$ profiles are of particular interest, owing to the presence of strong blue asymmetry (see Sect. 4.3), indicating possible infall motion (Leung \& Brown 1977; Zhou 1992; Gregersen et al. 1997). The spatial extent $(\sim 0.2 \mathrm{pc})$ of such an asymmetry is not inconsistent with the finding of Myers et al. 2000, who detected infall motion over a linear scale of $\sim 0.1 \mathrm{pc}$ in a large number of starless cores and Class 0/I protostars. However, it is hard to study the detailed infall structure with current spatial resolution. Contamination by rotation and outflows make a clearcut interpretation even more difficult. Further high spatial resolution observations are imperative to verify such a possible infall. 


\section{Conclusions}

We have presented detailed molecular line and sub-mm dust observations of the molecular cloud Lynds 870, a previously poorly studied region. The main results are:

1. Substantial molecular emission has been detected from Lynds 870. Two main cores, separated by $3^{\prime}$, are found both in dust (SMM1 and SMM2) and molecular line emission with the northern core (SMM1) being particularly rich in molecular lines. The continuum and spectroscopic observations indicate that the northern core, centered at IRAS $20231+3440$, is warmer and denser than the southern core (SMM2). A total mass of $\sim 70-110 M_{\odot}$ is estimated from the molecular line and the submillimeter dust emission.

2. Outflow activities are detected in both the northern and the southern core, indicative of on-going star formation in both regions. While IRAS 20231+3440 (IRS1 in $K_{\mathrm{s}}$ ) is found to be a Class I YSO candidate and most likely the exciting star of the northern outflow, a more deeply embedded companion (source 2 in $K_{\mathrm{s}}$ ) found next to IRAS 20231+3440 may also be a possible driving engine. The driving source of the southern outflow, still deeply embedded in the cloud, is most likely a Class 0 YSO candidate. The southern core (SMM2) is therefore still in an earlier evolutionary stage than the northern core (SMM1).

3. A significant amount of low velocity outflowing gas is hidden in the relatively broad systemic CO profiles tracing the quiescent gas. The low velocity outflow is, however, revealed by the $\mathrm{HCO}^{+}$emission. The total outflowing gas mass of the northern core is derived to be $8 M_{\odot}$.

4. Possible infall motions in the northern dense core are indicated by self-absorbed $\mathrm{HCO}^{+} J=3-2$ line profiles with a strong blue asymmetry; its spatial extent is $\sim 0.2$ pc.

5 . In a diameter of $8^{\prime}$ around IRAS $20231+3440$, there are in total $12 K_{\mathrm{s}}$ sources with a strong IR excess, typical of YSOs still embedded in their parental dust/molecular cloud. Combined with the outflows and $\mathrm{H}_{2} \mathrm{O}$ masers found in the region, we conclude that the cloud is presently actively forming a group or a small cluster of stars.

Acknowledgements. We appreciate the assistance of the HHT staff and Effelsberg-100m operators during the observations. We are grateful to Dr. D. Muders for his help in the reduction of HHT $870 \mu \mathrm{m}$ continuum data. We also benefited from useful discussions with Drs. Q. Zeng, G. Sandell, and Q. Zhang. We thank the referee whose helpful comments and suggestions greatly helped to improve the paper. This publication makes use of data products from the Two Micron All Sky Survey, which is a joint project of the University of Massachusetts and the Infrared Processing and Analysis Center, funded by the National Aeronautics and Space Administration and the National Science Foundation. R.Q.M. acknowledges support by the exchange program between the Chinese Academy of Sciences and the Max-Planck-Gesellschaft. This work was supported in part by grants 19625307 from NSFC \& G19990754 from CMST.

\section{References}

André, P., Ward-Thompson, D., \& Barsony, M. 1993, ApJ, 406, 122

André, P., Ward-Thompson, D., \& Barsony, M. 2000 in Protostar and Planets IV, ed. V. Mannings, A. P. Boss, \& S. S. Russell (Univ. Arizona Press, Tuscon), 59

Baars, J., Martin, R. N., Mangum, J. G., \& McMullin, J. P. 1999, PASP, 1111, 627

Bacmann, A., André, P., Puget, J.-L., Abergel, A., Bontemps, S., \& Ward-Thompson, D. 2000, A\&A, 361, 555

Blake, G. A., Masson, C. R., Phillips, T. G., \& Sutton, E. C. 1986, ApJS, 60, 357

Bohlin, R. C., Savage, B. D., \& Drake, J. F. 1978, ApJ, 224, 132

Bontemps, S., André, P., Terebey, S., \& Carbit, S. 1996, A\&A, 311,858

Brand, J., Cesaroni, R., Caseli, P., et al. 1994, A\&AS, 103, 541

Bronfman, L., Nyman, L.-A., \& May, J. 1996, A\&AS, 115, 81

Cabrit, S., \& Bertout, C. 1986, ApJ, 307, 313

Cabrit, S., \& Bertout, C. 1990, ApJ, 348, 530

Chin, Y., Henkel, C., Whiteoak, J. B., Langer, N., \& Churchwell, E. B. 1996, A\&A, 305,960

Danby, G., Flower, D. R., Valiron, R., Schilke, P., \& Walmsley, C. M. 1988 MNRAS, 235, 229

Downes, D. 1989, Evolution of Galaxies - Astronomical Observations, Lecture Notes in Physics 333, ed. I. Appenzeller, H. Habing, \& P. Lena (Springer Verlag, Berlin), 353

Draine, B. T., \& Lee, H. M. 1984, ApJ, 285, 89

Emerson, J. P. 1988, in Formation and Evolution of Low-mass Stars, ed. A. K. Dupree, \& M. T. V. T. Lago (Kluwer), 193

Frerking, M. A., Langer, W. D., \& Wilson, R. W. 1982, ApJ, 262,590

Fukui, Y., Iwata, T., Mizuno, A., Bally, J., \& Lane, A. P. 1993, Protostars \& Planets III, ed. E. H. Levy, \& J. I. Lunine (Tucson: Univ. of Arizona Press), 603

Furuya, R. S., Kitamura, Y., Wootten, H. A., Claussen, M. J., \& Kawabe, R. 2001, ApJ, 559, L143

Goodman, A. A., Benson, P. J., Fuller, G. A., \& Myers, P. C. 1993, ApJ, 406, 528

Gregersen, E. M., Evans, N. J. II, Zhou, S., \& Choi, M. 1997, ApJ, 484, 256

Groesbeck, T. G., Phillips, T. G., \& Black, G. A. 1994, A\&AS, 94,147

Harju, J., Walmsley, C. M., \& Wouterloot, J. G. A. 1993, A\&AS, 98, 51

Henning, T., Pfau, W., \& Altenhoff, W. J. 1990, A\&A, 227, 542

Henning, T., Cesaroni, R., Walmsley, M., \& Pfau, W. 1992, A\&AS, 93, 525

Hildebrand, R. H. 1983, QJRAS, 24, 267

Ho, P. T. P., \& Townes, C. H. 1983, ARA\&A, 21, 239

Hogerheijde, M. R., van Dishoeck, E. F., Blake, G. A., \& van Langevelde, H. J. 1997, ApJ, 489, 293

Hogerheijde, M. R., van Dishoeck, E. F., Salverda, J. M., \& Blake, G. A. 1999, ApJ, 513, 350

Irvine, W. M., Goldsmith, P. F., \& Hjalmarson, A. 1987, in Proc. IAU Symp. 120, Interstellar Processes, ed. D. J. Hollenbach, \& H. A. Thronson (Dordrecht:Reidel), 561

Jiang, Z., Wang, M., \& Yang, J. 2000, Acta Astron. Sinica, 41,28

Jijina, J., Myers, P. C., \& Adams, F. C. 1999, ApJS, 125, 161

Koornneef, J. 1983, A\&A, 128, 84 
Krügel, E., \& Siebenmorgen, R. 1994, A\&A, 288, 929

Lada, C. J., \& Adams, F. C. 1992, ApJ, 393, 278

Lada, C. J., Young, E. T., \& Greene, T. P. 1993, ApJ, 408, 471

Leung, C. M., \& Brown, R. L. 1977 ApJ, 214, L73

Loup, C., Forveille, T., Omont, A., \& Paul, J. F. 1993, A\&AS, 99, 291

Myers, P. C., Evans, N. J. II, \& Ohashi, N. 2000, in Protostar and Planets IV, ed. V. Mannings, A. P. Boss, \& S. S. Russell (Univ. Arizona Press, Tuscon), 217

Ossenkopf, V., \& Henning, T. 1994, A\&A, 291, 943

Palla, F., Cesaroni, R., Brand, J., et al. 1993, A\&A, 280, 599

Pickett, H. M., Poynter, R. L., Cohen, E. A., Delitsky, M. L., Pearson, J. C., \& Muller, H. S. P. 1998, J. Quant. Spectrosc. \& Rad. Transfer 60, 883

Preibisch, T., Ossenkopf, V., Yorke, H. W., \& Henning, T. 1993, A\&A, 279, 577

Sutton, E. C., Blake, G. A., Masson, C. R., \& Phillips, T. G. 1985, ApJS, 58, 341

Schilke, P., Groesbeck, T. D., Blake, G. A., \& Philips, T. G. 1997, ApJS, 108, 301

Scoville, N. Z., Sargent, A. I., Sanders, D. B., Claussen, M. J., Masson, C. R., Lo, K. Y., \& Phillips, T. G. 1986, ApJ, 303, 416

Shepherd, D. S., \& Churchwell, E. 1996, ApJ, 472, 225

Shepherd, D. S., Watson, A. M., Sargent, A. I., \& Churchwell, E. 1998, ApJ, 507, 861

Slysh, V. I., Dzura, A. M., et al. 1997, A\&AS, 124, 85
Stanek, K. Z., Knapp, G. R., Yonug, K., \& Phillips, T. G. 1995, ApJS, 100, 169

Walmsley, C. M., \& Ungerechts, H. 1983 A\&A, 122, 164

van der Walt, D. J., Retief, M. J., Gaylard, M. J., \& Macleod, G. C. 1996, MNRAS, 282, 1085

Visser, A. E., Richer, J. S., Chandler, C. J., \& Padman, R. 1998, MNRAS, 301, 585

Visser, A. E., Richer, J. S., \& Chandler, C. J. 2001, MNRAS, 323,257

Williams, J. P., Myers, P. C., Wilner, D. J., \& Di Francesco, J. 1999, ApJ, 513, 61

Wilson, T. L., \& Rood, R. T. 1994, ARA\&A, 32, 191

Wood, D. O. S., \& Churchwell, E. 1989, ApJS, 69, 831

Wu, Y., Huang, M., \& He, J. 1996, A\&AS, 115, 283

Yang, J., Jiang, Z., Wang, M., \& Wang, H. 1999, in Observational Astrophysics in Asia and its future, Proceedings of the 4th Asian meetings on Astronomy, ed. P. Chen (Kunming: Yunnan Astronomical Observatory), 80, 165

Yang, J., Jiang, Z. B., Wang, M., Ju, B. G., \& Wang, H. C. 2002, ApJS, in press

Zhang, Q., Hunter, T. R., Brand, J., Sridharan, T. K., Molinari, S., Kramer, M. A., \& Cesaroni, R. 2001, ApJ, 552,167

Zhou, S. 1992, ApJ, 394, 204

Zinchenko, I., Forsström, V., Lapinov, A., \& Mattila, K. 1994, A\&A, 288, 601 